\title{
GABA-glutamate supramammillary neurons control theta and gamma oscillations in the dentate gyrus during paradoxical (REM) sleep
}

\author{
Francesca Billwiller ${ }^{1}$. Laura Castillo ${ }^{2}$ Heba Elseedy ${ }^{2,4}$. Anton Ivanovich Ivanov ${ }^{2}$. Jennyfer Scapula ${ }^{2}$. \\ Antoine Ghestem ${ }^{2}$. Julien Carponcy ${ }^{1} \cdot$ Paul Antoine Libourel $^{1} \cdot$ Hélène Bras $^{3} \cdot$ Nabila ElSayed Abdelmeguid $^{4}$. \\ Esther Krook-Magnuson ${ }^{5} \cdot$ Ivan Soltesz $^{6} \cdot$ Christophe Bernard $^{2} \cdot$ Pierre-Hervé Luppi $^{1} \cdot$ Monique Esclapez $^{2}$ (D)
}

Received: 20 February 2020 / Accepted: 8 September 2020 / Published online: 24 September 2020

(c) The Author(s) 2020

\begin{abstract}
Several studies suggest that neurons from the lateral region of the SuM (SuML) innervating the dorsal dentate gyrus (DG) display a dual GABAergic and glutamatergic transmission and are specifically activated during paradoxical (REM) sleep (PS). The objective of the present study is to characterize the anatomical, neurochemical and electrophysiological properties of the SuML-DG projection neurons and to determine how they control DG oscillations and neuronal activation during PS and other vigilance states. For this purpose, we combine structural connectivity techniques using neurotropic viral vectors (rabies virus, AAV), neurochemical anatomy (immunohistochemistry, in situ hybridization) and imaging (light, electron and confocal microscopy) with in vitro (patch clamp) and in vivo (LFP, EEG) optogenetic and electrophysiological recordings performed in transgenic VGLUT2-cre male mice. At the cellular level, we show that the SuML-DG neurons co-release GABA and glutamate on dentate granule cells and increase the activity of a subset of DG granule cells. At the network level, we show that activation of the SuML-DG pathway increases theta power and frequency during PS as well as gamma power during PS and waking in the DG. At the behavioral level, we show that the activation of this pathway does not change animal behavior during PS, induces awakening during slow wave sleep and increases motor activity during waking. These results suggest that the SuML-DG pathway is capable of supporting the increase of theta and gamma power in the DG observed during PS and plays an important modulatory role of DG network activity during this state.
\end{abstract}

Keywords SuM $\cdot$ GAD $\cdot$ VGAT $\cdot$ VGLUT2 $\cdot$ Hippocampus $\cdot$ Hypothalamus

Francesca Billwiller, Laura Castillo and Heba Elseedy equal contributing first authors.

Pierre-Hervé Luppi and Monique Esclapez equal contributing senior authors.

Monique Esclapez

monique.esclapez@univ-amu.fr

1 UMR 5292 CNRS/U1028 INSERM, Centre hospitalier le vinatier, Neurocampus, University Lyon I, Bron, France

2 INSERM, INS, Institut de Neurosciences des Systèmes, Aix-Marseille Univ, Marseille, France

3 CNRS, INT, Institut de Neurosciences Timone, Aix-Marseille Univ, Marseille, France

4 Zoology Department, Faculty of Science, Alexandria University, Alexandria, Egypt

5 Department of Neuroscience, University of Minnesota, Minneapolis, MN 55455, USA

6 Department of Neurosurgery, Stanford University, Stanford, USA

\section{Introduction}

The supramammillary nucleus ( $\mathrm{SuM})$ is a thin structure overlying the mammillary bodies in the hypothalamus that provides substantial projections to many regions of the limbic system including the hippocampal formation (for review see Pan and McNaughton 2004; Vertes 2015). The SuM is composed of several populations of neurons that differ in their neurochemical content and the specificity of their connections (Swanson 1982; Gonzalo-Ruiz et al. 1992; Leranth and Kiss 1996; Kocsis et al. 2003; Borhegyi and Leranth 1997; Haglund et al. 1984). In rats, many studies have described the projections from SuM neurons to the hippocampus (Segal and Landis 1974; Pasquier and Reinoso-Suarez 1976, 1978; Wyss et al. 1979; Haglund et al. 1984; Saper 1985; Vertes 1992; Maglóczky et al. 1994; Nitsch and Leranth 1996; Vertes and 
McKenna 2000). We (Soussi et al. 2010) and others (Boulland et al. 2009) demonstrated in rats that SuM neurons from the most medial part of the SuM (SuMM; Paxinos and Watson 1998), referred as SuMm by Swanson (1998), or SuMp by Pan and McNaughton (2004) innervating the inner molecular layer of the ventral dentate gyrus (DG) and CA2 pyramidal cells are glutamatergic (Soussi et al. 2010). In contrast, SuM neurons located in the lateral twothird region of the SuM (SuML), referred as SuMg by Pan and McNaughton (2004), innervate the supragranular layer of the dorsal DG (dDG) and to a lesser extent the ventral DG (vDG) and display a unique dual glutamatergic and GABAergic neurotransmitter phenotype (Boulland et al. 2009; Soussi et al. 2010). Indeed, these SuML neurons and their projections to the $\mathrm{dDG}$ co-express markers for both glutamatergic (vesicular glutamate transporter 2; VGLUT2) and GABAergic (glutamate decarboxylase 65, GAD65 and vesicular GABA transporter, VGAT) neurotransmission and establish asymmetric and symmetric synapses on DG cells (Soussi et al. 2010). The connectivity and neurochemical properties of these different SuMhippocampal pathways suggest that they may contribute differently to hippocampal dependent functions. Indeed, it has been shown that the SuM controls hippocampal theta rhythm (Kocsis and Vertes 1994; Vertes and Kocsis 1997; Kocsis and Kaminski 2006) and is involved in emotional learning and memory (Pasquier and Reinoso-Suarez 1976; Richmond et al. 1999; Pan and McNaughton 2002; Santín et al. 2003; Pan and McNaughton 2004; Shahidi et al. 2004). By combining retrograde tracing, neurotoxic lesion and FOS immunostaining, it was recently shown that SuML GABA/glutamate neurons are responsible for the activation of dDG granule cells during paradoxical sleep (PS) (Renouard et al. 2015; Billwiller et al. 2017). Such activation may play a key role in the previously reported beneficial effect of PS on learning and memory (Maquet et al. 2000). In addition, Pedersen et al. (2017), by using a chemogenetic approach in transgenic mice, showed that SuM neurons containing only VGLUT2 but not those containing VGAT and VGLUT2 play a crucial role in waking. Altogether, these results suggest that glutamatergic neurons from the SuM known to innervate several limbic regions including the CA2/CA3a hippocampal region and the vDG are involved in normal wakefulness, whereas the GABA/ glutamate SuML neurons projecting to the $\mathrm{dDG}$ could be instrumental for PS function.

In this study we investigate how the SuML-dDG pathway controls dDG neuronal activity during PS and other vigilance states. For this purpose we combine innovative structural connectivity techniques using neurotropic viral vectors (rabies virus, AAV), neurochemical anatomy (immunohistochemistry, in situ hybridization) and imaging (light, electron and confocal microscopy) with in vitro (patch clamp) and in vivo (LFP, EEG) optogenetic and electrophysiological recordings performed in transgenic VGLUT2-cre mice in order: (1) to fully characterize the anatomical, neurochemical and electrophysiological properties of the SuML-dDG projection neurons in mice and (2) to determine the influences of this specific pathway on behaviors and associated oscillatory activities characterizing the different vigilance states as well as DG neuron activity.

\section{Materials and methods}

\section{Animals}

For this study, we used 26 transgenic "VGLUT2-cre" adult male mice (25-30 g; aged 10-11 weeks) obtained by mating male and female homozygote Vglut2-iresCre mice from Jackson Laboratories (strain name Slc17a6 ${ }^{\text {tm2(cre)Lowl } / J, ~ s t o c k ~ \# 016963) . ~ A l l ~ m i c e ~ w e r e ~ b r e d ~}$ in-house and maintained in standard cages, with food and water ad libitum, in a temperature- and humiditycontrolled room under a $12 \mathrm{~h}$ light/ $12 \mathrm{~h}$ dark cycle. All the surgical and experimental procedures were performed according to the National Institutes of Health guidelines and the European communities Council Directive of $86 / 609 / \mathrm{EEC}$ and were approved by the University of Aix-Marseille and Lyon University Chancellor's Animal Research Committees.

\section{Vectors}

\section{Rabies virus}

Four VGLUT2-cre mice underwent stereotaxic injection of rabies virus (RV) within the inner molecular layer of the $\mathrm{dDG}$ in order to obtain golgi-like retrograde-labeling of SuM neurons projecting to this region. The strain of RV used was the Challenge Virus Standard (CVS, $4.10^{7}$ plaque-forming units/mL) (Bras et al. 2008; Ugolini 2010; Coulon et al. 2011). Only vaccinated personnels conducted these experiments at the appropriate biosafety containment level until the sacrifice of the animals as described below.

These animals were used to determine the neurotransmiter phenotype of SuM neurons projecting to dDG at the 
mRNA level, combining immunohistofluorescent detection of RV with fluorescent in situ hybridization detection of VGAT and VGLUT2 mRNAs.

\section{Adeno-associated virus (AAV) double floxed inverted ORF (DIO) vectors}

Thirteen VGLUT2-cre mice underwent stereotaxic injection of the cre-dependent viral vectors: AAV5-EF1a-DIOEYFP $\left(3.5 \times 10^{12}\right.$ virus molecules/mL; UNC Gene Therapy Center Vector Core; Dr Deisseroth) into the SuML. These VGLUT2-EYFP mice expressing the Yellow Fluorescent Protein (YFP) in VGLUT2 SuM neurons and their axon terminals were used for the following: (1) to determine the neurotransmitter phenotype of SuM neurons innervating the $\mathrm{dDG}$ at the protein level, by simultaneous immunohistofluorescent detection of EYFP labeled axon fibers and terminals, VGAT and VGLUT2 in dDG $(n=3)$; (2) to determine the synaptic profile of these EYFP labeled axon terminals at the electron microscopy level $(n=3)$; (3) as control animals $(n=3)$ for in vitro optogenetic stimulation and patch clamp electrophysiological recordings; (4) as control animals $(n=4)$ for in vivo optogenetic stimulation and electrophysiological recordings followed by $\mathrm{cFos}$ immunolabeling.

Nine VGLUT2-cre mice underwent stereotaxic injection of the cre-dependent viral vectors: AAV5-EF1a-DIOhChR2(H134R)-EYFP $\left(3.2 \times 10^{12}\right.$ virus molecules $/ \mathrm{mL}$; UNC Gene Therapy Center Vector Core; Dr. Deisseroth) into the SuML. These VGLUT2-ChR2 mice expressing the excitatory opsin, channelrhodopsin 2 (ChR2) and the reporter protein EYFP in VGLUT2 SuM neurons and their axon terminals were used for (1) in vitro optogenetic stimulation and patch clamp electrophysiological recordings experiments $(n=5),(2)$ in vivo optogenetic stimulation and electrophysiological recordings followed by cFos immunolabeling $(n=4)$.

\section{Surgery}

Mice were anesthetized by an intraperitoneal injection (i.p.) of Ketamin $(50 \mathrm{mg} / \mathrm{kg}) / \mathrm{Xylazine}(5 \mathrm{mg} / \mathrm{kg})$ solution and additional injections were delivered as needed to maintain deep anesthesia during the surgery. Animals were then secured in a stereotaxic frame (David Kopf instruments). The body temperature of mice was monitored and maintained at about $37{ }^{\circ} \mathrm{C}$ during the entire procedure by means of an anal probe and heating blanket, respectively.
The head was shaved and sanitized with Betadine and 0.9\% $\mathrm{NaCl}$. Local anesthesia was performed by infiltration of the scalp with xylocaine (lidocaine hydrochloride $0.5 \%$ ), and an ophthalmic gel was placed on the eyes to avoid drying. After scalp incision, holes were drilled in the skull, with antero-posterior (AP), medio-lateral (ML) and dorsoventral (DV) coordinates based on Paxinos and Franklin's atlas (2005).

\section{$\mathrm{RV}$ and $\mathrm{AAV}$ viral vector injections}

The RV (200 nL) was pressure-injected unilaterally $(n=2)$ or bilaterally $(n=2)$ within the dDG inner molecular layer of VGLUT2-cre mice according to the following coordinates: $\mathrm{AP}=-2 ; \mathrm{ML}= \pm 1.5 ; \mathrm{DV}=-1.7$. Injections were performed by using a 33-gauge Hamilton syringe connected to a Micro4 injection pump system (World Precision Instruments). After completion of the injection procedures, the syringe was removed and the skin was sutured. Animals were treated with local anesthetic, returned to their cages kept at the appropriate biosafety containment level for a survival period of $38 \mathrm{~h}$ to observe an optimal RV retrograde labeling for the dendritic arbor of SuM neurons. This post-injection survival time was adjusted to obtain a Golgi-like labeling of the first-order infected neurons and to limit trans-neuronal spread to second order projection neurons, starting to be detected after $42 \mathrm{~h}$ (Fig. 1a-c). AAV5-EF1a-DIO viral vectors $(500 \mathrm{~nL})$ were injected bilaterally within the SuML of VGLUT2-cre mice at the following coordinates: $\mathrm{AP}=-2.7 ; \mathrm{LM}= \pm 1.25 ; \mathrm{DV}=-4.8$ using an $11^{\circ}$ angle to avoid the high vascularization at the midline.

\section{Optrode and electrode implantation}

After AAV injections, VGLUT2-mice were implanted with an optrode in the left $\mathrm{dDG}(\mathrm{AP}=-2 ; \mathrm{ML}=-1.1$; $\mathrm{DV}=-1.7$ ) for optic stimulation of axon terminals originating from transfected SuML neurons and local field potential (LFP) recordings from the DG. The optrode consisted of an optic fiber $(250 \mu \mathrm{m} \varnothing, 0.39 \mathrm{NA}$; Thorlabs SAS) and the LFP electrode. The LFP electrodes consisted of two $45 \mu \mathrm{m}$-diameter tungsten wires (California Fire Wire Company), twisted and glued together to form a rigid and solid structure, with the two ends of the wires separated by $100 \mu \mathrm{m}$ from each other (impedance 100 KOhms). Two small screws ( $1 \mathrm{~mm}$ in diameter, Plastics 

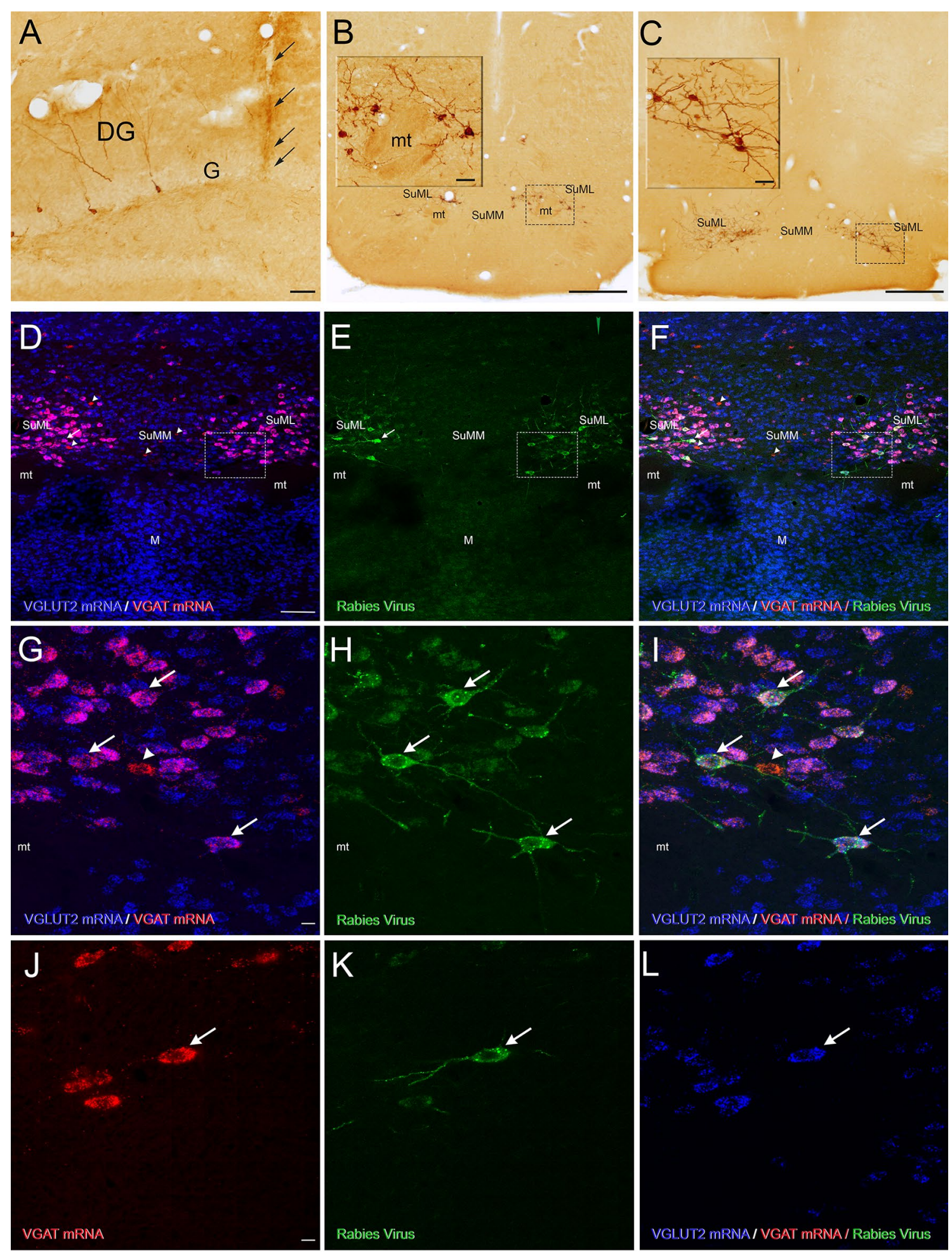
4Fig. 1 Neurochemical features of SuML neurons innervating the dorsal dentate gyrus (DG) characterized by simultaneous labeling for the rabies virus (RV) retrograde tracer (green), VGAT mRNA (red) and VGLUT2 mRNA (blue) in a coronal section. a-c Coronal sections of a VGLUT2-cre mouse processed for immunodetection of the $\mathrm{RV}, 38 \mathrm{~h}$ after its injection in supragranular and granule cell layers (G) of the dorsal DG. a Photomicrograph showing the track of the micropipette and site of injection (arrows). Sections at two rostro (b)-caudal (c) levels of the SuM showing retrograde-labeled neurons located within the lateral part of the SuM (SuML). Insert in b, $\mathbf{c}$ at higher magnification, Golgi-like labeling of the retrograde-labeled neurons. d-l All confocal images were obtained from sequential acquisition of separate wavelength channels, corresponding to the different fluorophores used for the triple labeling, from a single optical slice. This optical slice was acquired in the supramammillary region of the hypothalamus from a coronal section of a VGLUT2-cre mouse that received an injection of RV in supragranular layer of the dorsal DG (a). d Image obtained from the merge of the two confocal images corresponding, respectively, to the labeling of VGLUT2 (blue) and VGAT (red) mRNAs. Many neurons expressing both VGLUT2 and VGAT mRNAs (arrow) were observed in the lateral region of the supramammillary nucleus (SuML). They were located almost exclusively above and around the mammillary tract $(\mathrm{mt})$. Neurons expressing VGLUT2 mRNA only (blue) were observed mainly in the most medial part of the SuM (SuMM) and were numerous in the mammillary nucleus (M). Few neurons containing VGAT mRNA only (red, arrowheads) were distributed in the SuML and SUMM. e Confocal image corresponding to the immunohistochemical labeling of the $\mathrm{RV}$ (green) showing that all RV containing neurons in the SuM were located in the region of the SuML surrounded the mt. $\mathbf{f}$ Merge of $\mathbf{d}$, e. $\mathbf{g}-\mathbf{i}$ Higher magnification of region outlined in $\mathbf{d}-\mathbf{f}$ showing that all these SuML neurons projecting to the DG, labeled for the RV (h, arrows), co-expressed VGLUT2 and VGAT mRNAs (g, $\mathbf{i}$, arrows). $\mathbf{j}-\mathbf{l}$ RV labeled neuron ( $\mathbf{k}$, arrow) co-expressed VGAT mRNA (j, arrow) and VGLUT2 mRNA (l, arrow). Scale bars a $50 \mu \mathrm{m}$; b, c, $500 \mu \mathrm{m}$; insert, $50 \mu \mathrm{m} ; \mathbf{d}-\mathbf{f} 100 \mu \mathrm{m} ; \mathbf{g}-\mathbf{l} 10 \mu \mathrm{m}$

One) each soldered to a wire were fixed on the skull. The first screw was fixed in the parietal part of the skull for EEG recording and the second screw, at the level of cerebellum as reference electrode. Two gold-coated spherical electrodes were inserted in between neck muscles for differential EMG recording. All leads were connected to a miniature plug (Plastics One) that was cemented on the skull.

After completion of the surgery the skin was sutured. Mice were treated with local anesthetic, and an intramuscular injection of antibiotic (Baytril, $5 \mathrm{mg} / \mathrm{kg}$ ) to prevent any risk of infection. Mice were monitored until waking and returned to their home cages for a survival period of 3 weeks.

\section{Histology}

\section{Tissue preparation for light microscopy}

Animals were deeply anesthetized with ketamine and xylazine and transcardially perfused with $4 \%$ paraformaldehyde (PFA) in $0.12 \mathrm{M}$ sodium phosphate buffer, pH 7.4 (PB). After perfusion, the brains were removed from the skull, post-fixed in the same fixative for $1 \mathrm{~h}$ at room temperature (RT), rinsed in PB, cryoprotected in $20 \%$ sucrose overnight, frozen on dry ice and sectioned coronally at $40 \mu \mathrm{m}$ with a cryostat (Microm). The sections were rinsed in PB, collected sequentially in tubes containing an ethylene glycol-based cryoprotective solution and stored at $-20^{\circ} \mathrm{C}$ until histological processing. One of every ten sections was stained with cresyl violet to determine the general histological characteristics of the tissue throughout the rostro-caudal extent of the brain. Selected sections were processed for the following: (1) simultaneous detection of VGLUT2 mRNA, VGAT mRNA and RV; (2) simultaneous immunohistofluorescent detection of EYFP, VGLUT2 and GAD65 or VGAT; (3) immunohistochemical detection of cFos.

\section{Immunohistochemical detection of RV performed in VGLUT2-cre mice $(n=4)$ injected with RV}

Sections at the level of the DG and SuM were processed for immunohistochemical detection of the RV to identify the injection site and the retrograde-labeled neurons within the rostro-caudal extent of the SuM. Sections were pre-treated for $30 \mathrm{~min}$ in $1 \% \mathrm{H}_{2} \mathrm{O}_{2}$, rinsed in $\mathrm{PB}$ and in $0.02 \mathrm{M}$ potassium phosphate-buffered saline (KPBS, $\mathrm{pH}$ 7.2-7.4). Then, they were processed using a Mouse On Mouse kit (MOM, Vector Laboratories, Burlingame, CA, USA) to eliminate non specific labeling due to use of mouse monoclonal antibody on mouse tissue. Sections were incubated for $1 \mathrm{~h}$ at $\mathrm{RT}$ in MOM mouse IgG blocking reagent diluted in KPBS containing $0.3 \%$ Triton X-100. Sections were rinsed twice with KPBS for $5 \mathrm{~min}$ and pre-incubated in MOM diluent for $15 \mathrm{~min}$. They were then incubated overnight at RT in a solution containing the mouse monoclonal antibody directed against RV (1:3000; Raux et al. 1997; gratiously provided By Dr. Patrice Coulon), diluted in MOM diluent. After several rinses in KPBS, sections were incubated for $1 \mathrm{~h}$ in horse anti-mouse IgG (1:200, Vector Laboratories) diluted in MOM, rinsed in KPBS and incubated for $1 \mathrm{~h}$ at RT in an avidin-biotin-peroxidase complex solution prepared according 
to the manufacturer's recommendations (Vectastain $\mathrm{ABC}$ kit, Vector Laboratories). Sections were then processed for $15 \mathrm{~min}$ in 3.3'-diaminobenzidine tetrahydrochloride (DAB, Sigma fast tablets; Sigma), rinsed in KPBS, mounted onto Superfrost Plus slides, dehydrated and coverslipped with Permount.

\section{Simultaneous detection of VGLUT2 mRNA, VGAT mRNA and RV combining fluorescent in situ hybridization (RNAscope technology) and immunohistofluorescent methods performed in VGLUT2-cre mice $(n=4)$ injected with RV}

Selected sections at the level of the SuM were first treated with $1 \% \mathrm{H}_{2} \mathrm{O}_{2}$ rinsed in PB mounted on SuperFrost Plus slides (Fisher Scientific) and air dried at RT. They were then processed for fluorescent RNAscope in situ hybridization according to the manufacter's protocol (Advanced Cell Diagnostics). Briefly, sections were treated with $100 \%$ ethanol and protease III for $30 \mathrm{~min}$ at $40{ }^{\circ} \mathrm{C}$. They were incubated in a solution containing both RNAscope ${ }^{\circledR}$ ProbeMm-S1c17a6 for detection of VGLUT2 mRNA and MmS1c32a1-C3 for detection of VGAT mRNA. After hybridization, sections were processed for visualization using the RNA-scope Multiplex Fluorescent reagent Kit v2 (Advanced Cell Diagnostics) and the Tyramide Signal Amplification $\left(\mathrm{TSA}^{\mathrm{TM}}\right.$ ) Plus Cyanine 3 and TSA Plus Cyanine 5 systems (Perkin Elmer).

After the RNAscope assay, sections were rinsed in KPBS and processed for immnohistofluorescent detection of the $\mathrm{RV}$ using the MOM kit as described above. After the overnight incubation in the mouse monoclonal antibody directed against RV (1:3000), sections were rinsed in KPBS and incubated for $2 \mathrm{~h}$ in Alexa488-conjugated donkey antimouse (1:200; Invitrogen) diluted in MOM diluent. After several rinses in KPBS, all sections were coverslipped with Fluoromount (Electron Microscopy Sciences). The specimens were analyzed with a Zeiss laser-scanning confocal microscope.

\section{Quantification of co-localizing RV, VGLUT2 and VGAT mRNAs}

Quantitative analysis was conducted to evaluate the extent of SuM neurons with direct projections to the dDG that coexpress VGLUT2 and VGAT mRNAs. For this purpose, the number of triple-labeled neurons was determined for each animal $(n=4)$, from 3 sections $(120 \mu \mathrm{m}$ apart from each other) across the antero-posterior extent of the SuM. For each section, an image of the entire SuM region was obtained from a single confocal slice using the Tile Scan function with a $20 \times$ objective and sequential acquisition of the different wavelength channels to avoid fluorescent crosstalk with ZEN software (Zeiss). The analysis was then performed with Neurolucida software (version 7, mbfBioscience) as follows: for each confocal image, all RV-labeled neurons were identified on the green channels and examined for colocalization of VGLUT2 mRNA in the blue channel and/or VGAT mRNA in the red one. Triple-, double- and single-labeled neurons were tagged differently and counted by the software. A total of $380 \mathrm{RV}$-labeled neurons were analyzed.

\section{Simultaneous immunohistofluorescent detection of EYFP, VGLUT2 and GAD65 or VGAT performed in VGLUT2-EYFP mice $(n=3)$}

Selected sections at the level of the dDG were processed using the MOM kit as described above. Sections were incubated overnight at RT in a solution containing rabbit anti-GFP (1:2000; Invitrogen), guinea pig anti-VGLUT2 (1:5000; Millipore) and mouse anti-GAD65 (1:100; Millipore) or mouse anti-GFP (1:100; Invitrogen), guinea pig anti-VGLUT2 (1:5000; Millipore) and rabbit anti-VGAT (1:1000; Synaptic System) diluted in MOM diluent. After several rinses in KPBS, they were incubated for $2 \mathrm{~h}$ in Alexa488-conjugated donkey anti-rabbit IgG (1:200; Invitrogen), Cy5-conjugated donkey anti-guinea pig (1:100; Jackson ImmunoResearch Laboratories, Inc.), and Cy3conjugated donkey anti-mouse (1:100; Jackson ImmunoResearch Laboratories, Inc.) or Alexa488-conjugated donkey anti-mouse IgG (1:200; Invitrogen), Cy5-conjugated donkey anti-guinea pig (1:100; Jackson ImmunoResearch Laboratories, Inc.), and Cy3-conjugated donkey anti-rabbit (1:100; Jackson ImmunoResearch Laboratories, Inc.) diluted in MOM diluent. After several rinses in KPBS, all sections were then mounted on superfrost-coated slides, dried overnight at RT and coverslipped with Fluoromount. The specimens were analyzed with confocal microscope (Zeiss).

\section{Quantification of co-localizing GFP, VGLUT2 and VGAT}

Two quantification protocols were used in order to evaluate the extent of the different neurochemical phenotypes of axon terminals from the SuM innervating the dDG. Each of these two quantifications was obtained for each 
mouse $(n=3)$ from 4 sections $(400 \mu \mathrm{m}$ apart from each other) across the dDG. The first section was selected at rostral level when the granule cell and molecular layers start to form a "V" shaped structure enclosing the hilus corresponding to AP coordinate Bregma $-1.46 \mathrm{~mm}$ on Paxinos and Franklin's atlas (2005). The sections used in the first quantification protocol were adjacent to the sections used in the second protocol. In the first protocol previously described (Soussi et al. 2015), the densities of VGLUT2/VGAT and VGLUT2 only labeled terminals were assessed by the quantification of immunolabeling for VGLUT2/VGAT and VGLUT2 only, respectively. Single optical confocal images were acquired from sections $(n=4)$ of each mouse ( $n=3)$ with Zeiss LSM 510 laser-scanning microscope and analyzed with the software provided by the microscope manufacturer (LSM 510 Zen, Zeiss). All images were acquired from the suprapyramidal and infrapyramidal blades of the dDG, using identical parameters. The percentages of VGLUT2 labeled terminals containing VGAT were estimated by the Manders' coefficient (proportion of pixels for VGLUT2 also positive for VGAT) obtained with the JACoP co-localization Plugin for Image $\mathrm{J}$, in the region of interest (ROI) which included granule cell layer (GCL) and the narrow zone superficial to the granule cells defined as the supragranular layer (SGL) following recommendations from Bolte and Cordelières (2006). For each channel, an identical bottom threshold was applied throughout the analyses, and only the pixels with a value above this threshold were counted. When a pixel had a value above the threshold in both channels, it was counted as double positive. The size and the shape of the ROI was the same for each confocal image. The average $\%$ of co-localization was calculated for each blade of the DG for each mouse.

In the second quantification protocol, we determined, for each mouse $(n=3)$, the relative percentages of triple- and double-labeled boutons for the GFP-anterograde tracer and VGAT and/or VGLUT2 in the SGL of the dDG suprapyramidal and infrapyramidal blades. Several z-stacks of 10 confocal slices were acquired, from the different sections $(n=4)$, with a $100 \times$ objective and a numerical zoom of 8 , in each region of interest. The analysis was performed as previously described (Persson et al. 2006; Soussi et al. 2010) and following recommendations from Bolte and Cordelières (2006). For each z-stack, the confocal images obtained from separate wavelength channels (green, red and blue) were displayed side by side on the computer screen together with the images corresponding to colocalized pixels within each optical slice of the z-stack obtained with the colocalization highlighter plugin in ImageJ. The GFP-labeled boutons were identified in the green channel within a probe volume defined by the size of the confocal slice $(19.38 \mu \mathrm{m}$ by $19.38 \mu \mathrm{m})$ and the height of the $\mathrm{z}$-stack $(2 \mu \mathrm{m})$. Each bouton was examined for colocalization through the individual optical slices of the z-stack. Single-, double- and triple-labeled boutons were counted using the Cell Counter plugin in ImageJ. The total number of GFP-labeled terminals analyzed in the two regions of interest was 400 .

\section{Immunohistochemical detection of cFos performed in VGLUT2-EYFP $(n=4)$ and VGLUT2-ChR2 $(n=4)$ mice}

Selected sections at the level of the DG were processed for immunohistochemistry according to previously described protocol (Esclapez et al. 1994). Sections were pre-treated for $30 \mathrm{~min}$ in $1 \% \mathrm{H}_{2} \mathrm{O}_{2}$, rinsed in $\mathrm{PB}$ and KPBS, preincubated for $1 \mathrm{~h}$ in $3 \%$ normal goat serum (NGS, Vector Laboratories) diluted in KPBS containing 0.3\% Triton X-100 and incubated overnight at RT in cFos rabbit polyclonal antiserum (1:20,000; Calbiochem) diluted in KPBS containing $1 \%$ NGS and $0.3 \%$ Triton X-100. After several rinses in KPBS, sections were incubated for $1 \mathrm{~h}$ at RT in biotinylated goat anti-rabbit immunoglobulin $\mathrm{G}$ (IgG; Vector Laboratories) diluted 1:200 in KPBS containing 3\% NGS and then for $1 \mathrm{~h}$ at RT in an avidin-biotin-peroxidase complex solution prepared in KPBS according to the manufacturer's recommendations (Vectastain ABC kit, Vector Laboratories). Sections from VGLUT2-EYFP and VGLUT2-ChR2 mice were processed in parallel and for the same period of time (15 $\mathrm{min}$ ) in 3.3'-diaminobenzidine tetrahydrochloride (DAB, Sigma fast tablets; Sigma), rinsed in KPBS, mounted onto Superfrost Plus slides, dehydrated and coverslipped with Permount.

\section{Quantification of cFos immunolabeled neurons}

The number of cFos labeled neurons was calculated in the DG GCL of the right and left (ipsilateral to the optic stimulation) hemispheres in VGLUT2-EYFP control $(n=4)$, and VGLUT2-ChR2 $(n=4)$ mice. These analyses were performed using a computer-assisted system connected to a Nikon 90i microscope and the Neurolucida software (MicroBrightField). A total of 4 sections $(400 \mu \mathrm{m}$ apart from each other) surrounding the optrode site were analyzed for each animal. In each section the GCL was 
delineated and all neurons labeled for cFos were plotted. The number of labeled neurons, obtained from the 4 sections, was calculated in each hemisphere for each animal. The average total number of labeled neurons/hemisphere \pm SEM was calculated for each group of control VGLUT2-EYFP and VGLUT2-ChR2 mice. Statistical analysis was performed by Statview software using Wilcoxon Rank Sum Test.

\section{Tissue preparation for electron microscopy}

Three VGLUT2-EYFP mice were perfused intracardially with a fixative solution containing $4 \%$ PFA and $0.1 \%$ glutaraldehyde in $0.12 \mathrm{~m} \mathrm{~PB}$. After perfusion, the brain was removed from the skull, post-fixed in the same fixative overnight at $4{ }^{\circ} \mathrm{C}$ and rinsed in PB for $1.5 \mathrm{~h}$. Blocks of the forebrain were sectioned coronally at $60 \mu \mathrm{m}$ with a vibratome. Pre-embedding immunolabeling for GFP was performed on sections at the level of dDG. These sections were pre-treated for $15 \mathrm{~min}$ in $1 \%$ sodium borohydride prepared in PB and rinsed for $30 \mathrm{~min}$ in $\mathrm{PB}$ and $3 \times 30 \mathrm{~min}$ in KPBS. They were incubated for $1 \mathrm{~h}$ in normal goat serum diluted in $0.02 \mathrm{M}$ KPBS, then overnight in primary antibody rabbit anti-GFP (1:2000) diluted in KPBS containing normal goat serum at RT. On the following day, sections were rinsed for $1.5 \mathrm{~h}$ in $0.02 \mathrm{M} \mathrm{KPBS}$ then incubated for $1 \mathrm{~h}$ in the secondary antibody goat anti-rabbit (1:200) diluted in KPBS containing normal goat serum. After rinsing in KPBS for $1.5 \mathrm{~h}$, sections were incubated for $1 \mathrm{~h}$ in an avidin-biotinylated-peroxidase complex (ABC Elite; Vector Laboratories) prepared in KPBS. After $3 \times 30 \mathrm{~min}$ rinses in KPBS, sections were incubated for $12 \mathrm{~min}$ in 3.3'-diaminobenzidine tetrahydrochloride and $0.01 \% \mathrm{H}_{2} \mathrm{O}_{2}$, rinses in KPBS, post-fixed in 2\% PFA and 2.5\% Glutaraldahyde diluted in PB for $3 \mathrm{~h}$, then washed for $1.5 \mathrm{~h}$ in PB. After all these steps, sections were treated with $1 \%$ osmium tetroxide in PB for 45 min, dehydrated in ethanol, flat embedded in Durcupan resin and polymerized at $56^{\circ} \mathrm{C}$ for $24 \mathrm{~h}$ (Zhang and Houser 1999). Labeled regions of the DG that contained the molecular and granule cell layers were trimmed from the sections, re-embedded on capsules filled with polymerized Durcupan and further polymerized at $56{ }^{\circ} \mathrm{C}$ for an additional $24 \mathrm{~h}$. Ultrathin sections from the most superficial face of the blocks were cut on an ultramicrotome. Serial sections were picked up on nickel mesh grids and stained with uranyl acetate and lead citrate. Sections were examined and photographed with a JEOL electron microscope.

\section{In vitro electrophysiology: optic stimulation and patch clamp recordings}

\section{Hippocampal slice preparation}

VGLUT2-ChR2-EYFP mice $(n=5)$ and VGLUT2-EYFP mice $(n=3)$ were decapitated under isofluorane anesthesia. Brains were quickly removed and placed into an ice-cold $\left(4{ }^{\circ} \mathrm{C}\right)$ cutting solution containing (in $\mathrm{mM}$ ): 140 potassium gluconate, 10 HEPES, 15 sodium gluconate, 0.2 EGTA, $4 \mathrm{NaCl}$ ( $\mathrm{pH} 7.2$ ). Coronal slices were cut $(350 \mu \mathrm{m})$ using a vibratome (Leica Microsystem). In order to increase cell survival over time in slices from 10- to 11 week-old adults, slices were incubated first in a solution containing (in $\mathrm{mM}$ ): 110 choline chloride; 2.5 $\mathrm{KCl} ; 1.25 \mathrm{NaH}_{2} \mathrm{PO}_{4} ; 10 \mathrm{MgCl}_{2}, 0.5 \mathrm{CaCl}_{2} ; 25 \mathrm{NaHCO}_{3}$; 10 glucose, 5 sodium pyruvate, for $15 \mathrm{~min}$ at $20-23{ }^{\circ} \mathrm{C}$. Then, they were transferred to a holding chamber containing an artificial cerebrospinal fluid (ACSF) composed of (in $\mathrm{mM}$ ) $126 \mathrm{NaCl}, 3.5 \mathrm{KCl}, 1.2 \mathrm{NaH}_{2} \mathrm{PO}_{4}, 1.3 \mathrm{MgCl}_{2}, 2$ $\mathrm{CaCl}_{2}, 25 \mathrm{NaHCO}_{3}, 10$ D-glucose (pH 7.3-7.4) at RT for at least $1 \mathrm{~h}$ before recording. The two last solutions were saturated with $95 \% \mathrm{O}_{2}$ and $5 \% \mathrm{CO}_{2}$.

\section{Whole-cell voltage-clamp recordings}

Slices were submerged in a low-volume recording chamber and continuously superfused with $32-34{ }^{\circ} \mathrm{C}$ ACSF at $5 \mathrm{~mL} / \mathrm{min}$ perfusion rate. For each mouse, four slices containing the $\mathrm{dDG}$ were selected for patch clamp recordings. DG neurons were visualized by infrared video microscopy using an upright microscope (SliceScope, Scentifica Ltd). Patch pipettes were pulled from borosilicate glass tubing ( $1.5 \mathrm{~mm}$ outer diameter, $0.5 \mathrm{~mm}$ wall thickness) and filled with an intracellular solution containing (in mM) $20 \mathrm{CsCl}, 115$ CsGlu, 10 HEPES, 1.1 EGTA, 4 MgATP, $10 \mathrm{Na}$ phosphocreatine and $0.4 \mathrm{Na}_{2} \mathrm{GTP}$ as well as $0.2 \%$ biocytin for post-hoc morphological identification of the recorded neuron (see below). The pipette resistance was 4-6 M $\Omega$. Recordings were performed in the apex, suprapyramidal and infrapyramidal blades of the dDG. Signals were fed to a Multiclamp 700A (Molecular Devices), digitized $(10 \mathrm{kHz})$ with a DigiData 1550 (Molecular Devices) interface to a personal computer and analyzed with ClampFit software (Molecular Devices). Optical stimulation of ChR2-expressing axon terminals was performed by pulses of $470 \mathrm{~nm}$ blue light delivered by a LED (pE-2, CoolLED) through a $40 \times$ objective attached to microscope 
(SliceScope, Scientifika Ltd). Stimulations consisted of paired $5 \mathrm{~ms}$ pulses $(500 \mathrm{~ms}$ between pulses, every $30 \mathrm{~s})$. For VGLUT2-ChR2 mice, the light intensity corresponded to $20-30 \%(1.6-2.5 \mathrm{~mW})$ of the LED maximum power (7.5 $\mathrm{mW}$ ) and for the VGLUT2-EYFP control mice, it varied between 20 and $90 \%(6.9 \mathrm{~mW})$ of LED maximum power. Postsynaptic current (PSC) responses to optic stimulations were recorded at different holding potentials ranging from - $70 \mathrm{mV}$ (close to reversal potential of GABA-A currents but far below the reversal potential of glutamate receptormediated currents) to $+10 \mathrm{mV}$ (close to reversal potential of glutamate receptor-mediated currents but far above the reversal potential of GABA-A-mediated currents). Pharmacological characterization of inhibitory PSCs (IPSCs) and excitatory PSCs (EPSCs) was achieved using antagonists of GABA-A, AMPA and NMDA receptors. We used Gabazine or bicuculline (antagonist of GABA-A receptors, $10 \mu \mathrm{M}$ ), D-AP5 (antagonist of NMDA receptors, $40 \mu \mathrm{M}$ ) and NBQX (antagonist of AMPA and Kainate receptors, $10 \mu \mathrm{M}$ ). The co-release of glutamate and GABA was further demonstrated in several DG granule cells $(n=5)$, by recording first light stimulated PSC at $-70 \mathrm{mV}$ (Fig. 4k). At this holding potential the recorded currents were essentially generated by glutamate receptors. Then the glutamate component was abolished by NBQX and D-AP5, and the GABA component was revealed at $+10 \mathrm{mV}$ holding potential. This PSC was completely inhibited by bicuculline application that confirmed the GABA-A receptor origin of these remaining currents (see also Fig. 4j).

\section{Double immunohistofluorescent labeling for Biocytin and GFP}

After recordings, slices were processed for simultaneous detection of the biocytin-filled neurons and GFPlabeled axon fibers and terminals in order to identify the recorded cells and evaluate the efficiency of the transfection, respectively. Slices were fixed overnight at $4{ }^{\circ} \mathrm{C}$ in a solution containing 4\% PFA in PB. Then they were rinsed in $\mathrm{PB}$, cryoprotected in $20 \%$ sucrose and quickly frozen on dry ice. After several rinses in KPBS, slices were incubated in a solution containing normal donkey serum (NDS, 1:30; Vector Laboratory) diluted in KPBS with $0.3 \%$ Triton- X100, for $2 \mathrm{~h}$ at RT. They were incubated in a solution containing goat anti-biotin (1:200) and rabbit anti-GFP (1:2000), diluted in KPBS containing $0.3 \%$ Triton-X100 and NDS (1:100), overnight at RT.
After several rinses in KPBS, slices were incubated for $2 \mathrm{~h}$ in Alexa488-conjugated donkey anti-goat $\operatorname{IgG}(1: 200$; Invitrogen), and Cy3-donkey anti-rabbit $\operatorname{IgG}(1: 100)$ diluted in KPBS with $0.3 \%$ Triton-X100. After rinses in KPBS, slices were mounted on slides and coverslipped with Fluoromount. The specimens were analyzed with a fluorescence microscope (Nikon 50i) or confocal microscope (Zeiss).

\section{In vivo electrophysiology: optic stimulation, LFP and EEG recordings}

All mice were placed for 7 days in a recording box in order to get them used to the recording conditions. The recording box was ventilated, as well as electrically and sound isolated. The temperature was regulated at $21^{\circ} \mathrm{C}$, and a $12 \mathrm{~h}$ light $/ 12 \mathrm{~h}$ dark cycle imposed. Mice were accustomed to the cable connecting them to the recording device. The recording cable connected the micro-connector implanted on the head of the animal to a collector, which ensured the continuity of the recorded signals without hindering the movements of the mouse. At the end of this habituation, the control recordings begun. EEG and EMG recordings were digitized at $1 \mathrm{kHz}$, amplified 5000 times with a 16-channel amplifier (A-M System) and collected on a computer via a CED interface using Spike 2 software (Cambridge Electronic Design). The signal was band-pass filtered online between 1 and $300 \mathrm{~Hz}$ for EEG, and between 10 and $100 \mathrm{~Hz}$ for EMG. The $50 \mathrm{~Hz}$ signal was removed with a notch filter. The EEG and LFP signals were acquired by monopolar derivation (differential between the recording electrode and the reference electrode located above the cerebellum). The EMG bipolar signals were calculated by measuring the differential between the two EMG electrodes. Mice were recorded for $24 \mathrm{~h}$ of baseline followed by optogenetic manipulation.

\section{In vivo optogenetic stimulation}

Optical stimulations were delivered via a patch cable connected to a $100 \mathrm{~mW} 473$-diode (Laserglow). Stimulations were performed during 4 days: in the first 3 days, mice were stimulated during one specific vigilance state par day: waking (WK), slow wave sleep (SWS) or PS. Each day, stimulations were delivered during the same circadian period (10 AM-2 PM). Stimulations were applied $10 \mathrm{~s}$ after the occurrence of a stable WK, SWS or PS event as detected 
by real-time observation by the experimenter. For WK and SWS, stimulations were spaced apart by at least $1 \mathrm{~min}$ and for PS, by at least $15 \mathrm{~s}$.

Blue exciting stimulations consisted of 10 -s trains of 10 -ms pulses at $20 \mathrm{~Hz}$. Light power at the fiber tip was 10 $\mathrm{mW}$.

The 4th day of experiments, 4 VGLUT2-EYFP and 4 VGLUT2-ChR2 animals were stimulated for $15 \mathrm{~min}$ at $20 \mathrm{~Hz}$ (10-ms pulses). All mice were killed 90 min after the beginning of the stimulation by transcardiac perfusion of $4 \%$ PFA. Brain tissues were processed for immunohistochemical detection of cFos expression (see above).

\section{Analysis of the sleep wake states}

Polysomnographic recordings were visually scored by 5 -s epochs for WK, SWS and PS as previously described (Sapin et al. 2009). Hypnograms were obtained by using a custom Matlab script. For each animal, the number of awakenings during SWS and PS optogenetic stimulations was counted and expressed as percentage of the total number of stimulations.

\section{LFP and EEG analysis}

LFP and EEG signals were analyzed using a custom Matlab script using the Chronux toolbox. The time-frequency spectrograms were computed with the same toolbox and expressed in arbitrary units. The mean power spectral density in the $10 \mathrm{~s}$ before the stimulation was compared to that in the $10 \mathrm{~s}$ during the stimulation (sliding window: $1 \mathrm{~s}$ ), in order to obtain a mean spectral power ratio $(\mathrm{PR}) \pm \mathrm{SEM}$ for frequencies equal or above $1 \mathrm{~Hz}$. The frequency spectra were grouped into frequency bands commonly used Delta: 1-4 Hz, Theta: 6-12 Hz, Sigma: 12-14 Hz, Beta: 15-30 Hz, Gamma: 30-100 Hz. Power spectral values at $20 \mathrm{~Hz}$ and its harmonics were excluded from the analysis.

To analyze the evolution of LFP and EEG theta and gamma bands during optogenetic stimulation, the mean PR of these spectral bands and the respective $95 \%$ confidence intervals were calculated from $10 \mathrm{~s}$ before to $10 \mathrm{~s}$ after the photostimulation. In order to compute these intervals, we used a bootstrap procedure, which allows creating artificial groups from the original data, with replacement. The mean of each artificial group derived from the original data was then computed. This operation was repeated 10,000 times and the $95 \%$ confidence interval was the 5 th and the 95 th percentile of the means of the randomly constructed samples. Finally, during WK and PS the peak of theta frequency $(6-12 \mathrm{~Hz})$ in the $10 \mathrm{~s}$ before and during the optogenetic stimulation was identified in all animals.

Analysis of variance (Mann-Whitney test) was performed on the percentage of awakenings, the mean spectral power ratios and the theta frequency peaks. These statistics were performed using Statview software (StatView Inc, Nestbit, NS).

\section{EMG analysis}

EMG signals during WK were analyzed using a custom Matlab script. The mean EMG value in the $10 \mathrm{~s}$ before the stimulation was compared to that in the $10 \mathrm{~s}$ during the stimulation, in order to obtain a mean EMG ratio \pm SEM. In the two groups of animals we performed a sequential analysis per $0.5 \mathrm{~s}$ on the mean of the absolute EMG values from $20 \mathrm{~s}$ before to $10 \mathrm{~s}$ after the photostimulation. The respective bootstrap 95\% interval (computed as described above) was calculated for each sequential value.

Further, analysis of variance (Mann-Whitney test) was performed on the EMG mean by using Statview software.

\section{Results}

\section{Distribution of VGAT and/or VGLUT2 mRNA-containing neurons in the SuM of VGLUT2-Cre transgenic mice}

Three populations of neurons were observed at all anteroposterior levels of the SuM. These included a population of neurons co-expressing VGAT and VGLUT2 mRNAs, a population of neurons containing VGAT mRNA only (Fig. 1d, $\mathrm{g}$, arrowheads) and one expressing VGLUT2 mRNA only (Fig. 1d, g, blue). The cell body size, as well as the distribution and density, within the SuM, of these three populations of neurons differed significantly. Almost all VGAT/ VGLUT2 mRNAs-containing neurons displayed a large cell body $(30 \mu \mathrm{m})$ and were clustered around and above the mammillary tract (mt) (Fig. 1d, g) in a region similar to that described in rats as the grandicellular SuM (SuMg) by Pan and Mc Naughton (2004) and that is included in the lateral SuM (SuML) region of Paxinos and Franklin's mouse Atlas. The numerous singly labeled VGLUT2 mRNA -expressing cells were small $(10-15 \mu \mathrm{m})$ and mostly located in the most 
central area (Fig. 1d) termed the parvicellular SuM (SuMp) by Pan and McNaughton (2004) corresponding to the SuMm in Paxinos and Franklin's mouse Atlas. Some were also intermingled with the VGAT/VGLUT2 mRNAs-containing cells in the SuMg. Few neurons expressed VGAT mRNA only. These neurons displayed a small cell body $(15 \mu \mathrm{m})$ and were scattered within the SuML and SuMM.

In summary, our results indicate that in mice like in rats a large number of neurons co-expressing markers of GABAergic and glutamatergic transmission are located in the SuML region.

\section{Distribution of SuM neurons with dorsal dentate gyrus (dDG) projections}

Unilateral or bilateral injections of rabies-virus (RV) were performed into the DG of the dorsal hippocampal formation (Fig. 1a) to evaluate the distribution of SuM neurons that project to this structure. In all these mice, the RV tracer injection was located either in the granule cell layer or inner molecular region of the suprapyramidal blade of the DG. All these animals displayed retrogradely labeled neurons that were located exclusively in the region of SuML (SuMg) throught the rostro (Fig. 1b, e, h) —caudal (Fig. 1c) extent of the SuM. Virtually none were found in the SuMM (Fig. 1b, c, e). Many of these neurons displayed a large soma with several labeled proximal dendrites (Fig. 1b, c, e). Therefore, in mice SuM neurons innervating the $\mathrm{dDG}$ are located in the SuML as in rats (Soussi et al. 2010).

\section{Neurotransmitter phenotype of SuM neurons projecting to the $\mathrm{dDG}$ revealed by simultaneous detection of $R V$ retrograde tracer, VGAT $M R N A$, and VGLUT2 mRNA}

We next investigated whether the SuML neurons projecting to the dDG express markers of GABAergic and glutamatergic transmissions. Sections processed for simultaneous detection of VGAT mRNA, VGLUT2 mRNA and RV (Fig. 1d-l) showed that almost all RV retrogradely labeled neurons co-express VGAT and VGLUT2 mRNAs (Fig. 1d-1, arrows). These data were confirmed by quantitative analysis performed on 12 sections ( 4 mice; 3 sections per mouse) showing that $99 \%$ ( $n=378$ out of 380 neurons) of the
RV-labeled neurons co-expressed VGAT and VGLUT2 mRNAs. The percentages of these triple-labeled cells were the same (99\%) for the three antero-posterior levels of the SuM analyzed (range $98-100 \%$ ).

\section{Distribution and neurotransmitter phenotype of fibers and axon terminals originating from SuM neurons innervating the dorsal dentate gyrus}

To further characterize the neurochemical properties of SuML neurons innervating the dDG, AAV-5-DIO-EYFP was injected bilaterally in the SuML (Fig. 2a) allowing specific EYFP labeling of VGLUT2 neurons and of their axon fibers innervating the hippocampus including the $\mathrm{dDG}$ (Fig. 2a). Sections of these VGLUT2-EYFP mice were processed for simultaneous immunohistofluorescent detection of EYFP, VGLUT2 and VGAT or GAD65. All mice injected in the SuML displayed numerous anterograde-EYFP labeled fibers and axon terminals in the dDG (Fig. 2b, arrowheads) on both sides of the hippocampal formation. Labeled fibers and axon terminals were also present in the CA2/CA3a region of the hippocampus in particular when the injection sites involved more posterior levels of the SuML (Fig. 2b, arrowhead).

In the dDG, EYFP axonal fibers and terminals were mainly located in the supragranular layer of the suprapyramidal and infrapyramidal blades of the DG (Fig. 2b-d) although some were localized in the granule cell layers. In all triple labeled sections either for EYFP, GAD65 and VGLUT2 or EYFP, VGAT and VGLUT2, the vast majority if not all EYFP-containing axon terminals present in the supragranular and granule cell layers were labeled for both GAD65 and VGLUT2 (Fig. 2c, e-h) or VGAT and VGLUT2 (Fig. 2d, i-1). Quantitative analysis of double labeling for VGLUT2 and VGAT showed that 90\% (range 82-99\%) of VGLUT2-labeled terminals were labeled for VGAT with no major differences between the infrapyramidal blade (89\%; range $82-99 \%$ ) and the suprapyramidal blade (91\%; range 83-99\%). Further quantification of EYFP labeling revealed that $98 \%$ (range $96 \%$ and $100 \%$ ) of the axon terminals contained both VGAT and VGLUT2 confirming that all EYFP-containing axon terminals originating from SuML neurons contained both markers of GABA and glutamate neurotransmissions. 

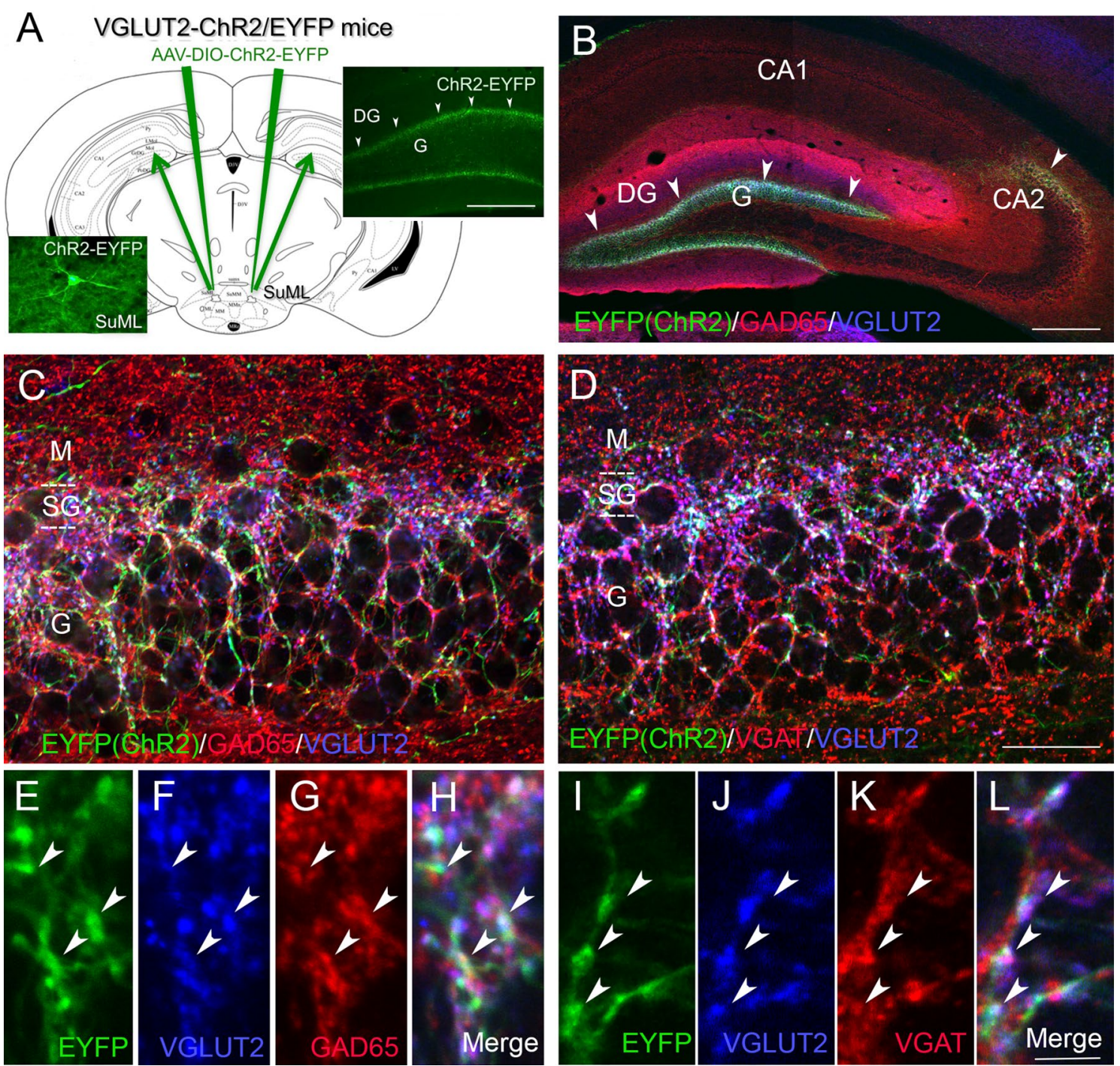

Fig. 2 Neurochemical features of axon terminals from SuML neurons innervating the dorsal DG characterized by simultaneous immunohistochemical labeling for the AAV-EYFP anterograde tracer (green), GAD65 or VGAT (red) and VGLUT2 (blue) in coronal sections. a Diagram illustrating the bilateral injections of the viral vector AAV-DIO-ChR2-EYFP within the lateral region of the SuM. Images from a coronal section showing endogenous fluorescence of EYFP observed in the cell body and proximal dendrites of a transfected neuron located within the SuML as well as fibers and axon terminals within the supragranular (arrowheads) and granular layer (G) of the DG. b Image obtained from a single optical slice showing labeling for EYFP (green), GAD65 (red) and VGLUT2 (blue) in the hippocampus. Fibers labeled with the anterograde tracer AAVDIO-ChR2-EYFP injected within the SUM as illustrate in a were exclusively located in the supragranular (arrowheads) and granule cell $(\mathrm{G})$ layers as well as the CA2 region of the hippocampus. c, d Images corresponding to a maximum intensity z-projection of a stack

of 8 optical slices spaced at $370 \mathrm{~nm}$, showing labeling for EYFP (green), VGLUT2 (blue) and GAD65 (c, red) or VGAT (d, red) in the dorsal DG. Axon terminals and fibers, from neurons in the SuML, labeled for the EYFP anterograde tracer (green) were located mainly in the supragranular layer but also in the granule cell layer. Numerous GAD65- (c) or VGAT- (d) containing terminals were present in the molecular layer (M) and granule cell layer (G) of the dorsal DG. VGLUT2-containing terminals were mainly located in the supragranular layer (SG) but were also observed in G. e-l Images of the three different fluorophores used for the triple labeling, obtained by sequential acquisition of separate wavelength channels from a single optical slice, in the SG of the DG demonstrated that many if not all axon terminals labeled for EYFP (b, i, green, arrowheads) contained GAD65 (g, red arrowheads), VGAT (k, red, arrowheads) but also VGLUT2 (f, $\mathbf{j}$ blue, arrowheads). h Merge of $\mathbf{e}-\mathbf{g}$. 1 Merge of $\mathbf{i}-\mathbf{k}$. Scale bars $\mathbf{a}, \mathbf{b}$, $200 \mu \mathrm{m} ; \mathbf{c}, \mathbf{d}, 25 \mu \mathrm{m}$ and $\mathbf{e}-\mathbf{l}, 3 \mu \mathrm{m}$ 

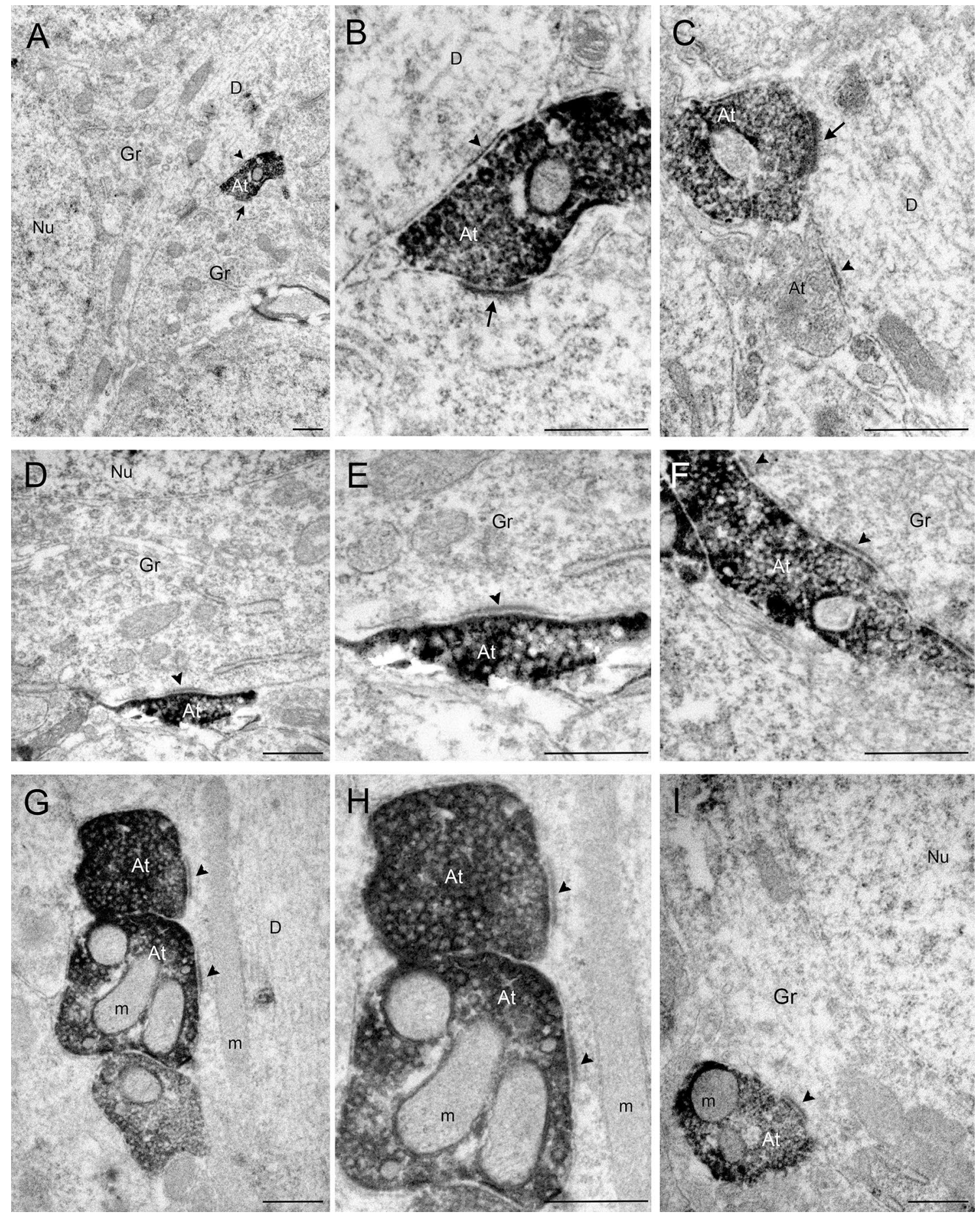

Fig. 3 Pre-embedding immunolabeling for EYFP anterograde tracer in ultrathin coronal sections of the dorsal DG. a-i In the dorsal DG, numerous axon terminals were labeled for EYFP, revealed by electron-dense peroxidase 3.3'-diaminobenzidine tetrahydrochloride product. a A labeled axon terminal (At) making two synaptic contacts (arrow and arrowhead) on unlabeled somata of 2 presumed granule cells (Gr). b Higher magnification of the At illustrated in a showing that these two synaptic contacts were different: one displayed a relatively thin post-synaptic density (arrowhead) characteristic of sym- metric synapses on the soma of one Gr; the other one displayed a thick post-synaptic density (arrow) characteristic of asymmetric synapses on the soma of another Gr. c Labeled axon terminal established a synaptic contact displaying a thick synaptic density on a unlabeled dendritic process (D) that differed from the synaptic contact formed by the unlabeled At (arrow). The synaptic contacts illustrated in $\mathbf{d}-\mathbf{i}$ displayed relatively thin post-synaptic densities (arrowheads) on the soma $(\mathbf{d}-\mathbf{f}, \mathbf{i})$ or dendrites $(\mathbf{g}, \mathbf{h})$ of unlabeled Gr. $m$ mitochondria, $\mathrm{Nu}$ nucleus. Scale bar $\mathbf{a}-\mathbf{i} 0.5 \mu \mathrm{m}$ 


\section{Electron microscopy analysis of synaptic contacts established by EYFP-containing axon terminals originating from SuML neurons}

In the supragranular region of the $\mathrm{dDG}$, axon terminals from SuML neurons labeled for EYFP displayed diffuse electrondense labeling that contrasted with adjacent unlabeled cellular compartments. These labeled axon terminals formed synaptic contacts on the soma (Fig. 3a, b, d, e, f, i) and dendritic profiles (Fig. 3c, g, h) of presumed granule cells (GCs). These axon terminals were often very large boutons that displayed one (Fig. 3a, b, d, e) or more (Fig. 3f) synaptic zones with relatively thin post-synaptic densities (arrowheads) characteristic of symmetric synapses. Other synaptic contacts formed by these labeled terminals displayed thicker post-synaptic densities characteristic of asymmetric synapses (Fig. 3a-c; arrow). We also observed large labeled "en passant" boutons establishing symmetric synapses on the dendrites of a presumed granule cell (Fig. 3g, h). Finally, some axon terminals from SuML neurons formed both symmetric (arrowhead) and asymmetric (arrow) synapses on the soma or the proximal dendrite of two different neighboring GCs (Fig. 3a, b).

Together these data demonstrate that in mice all SuM neurons innervating the $\mathrm{dDG}$ belong to a single population of large neurons located in the SuMg region of the SuML. These cells display a dual neurochemical phenotype for GABA and glutamate neurotransmissions and establish symmetric (presumably inhibitory) and asymmetric (presumably excitatory) synapses on the GCs of the dDG.

\section{Co-release of glutamate and GABA at the SuML-dentate granule cells synapses}

The neurochemical profile of SuML neurons projecting to the dDG strongly suggested that they co-release GABA and glutamate at the SuML-dentate granule cells synapses. To test this hypothesis, we performed patch clamp recordings of dDG granule cells layer using optogenetic stimulation in hippocampal slices obtained from VGLUT2-ChR2 mice (5 mice, 3 sections per mouse, 16 neurons) and VGLUT2-EYFP control mice (3 mice, 3 sections per mouse, 10 neurons) (Fig. 4). The light stimulation of SuML-DG fibers and axonal terminals expressing ChR2 (Fig. 4a) evoked a fast inward and a slower outward synaptic current in fourteen out of 16 neurons recorded at a holding potential of $-30 \mathrm{mV}$ (Fig. 4d, f, h). Post-hoc immunodetection of biocytin-filled neurons showed that all these recorded neurons correspond to GCs (Fig. 4c, e, $\mathrm{g}, \mathrm{i})$. They were equally distributed in the apex and the suprapyramidal and infrapyramidal blades of dDG granule cell layer
Fig. 4 Selective stimulation of axonal terminals from SuML neurons innervating the dorsal DG, performed in hippocampal slices of VGLUT2-ChR2-EYFP mice, induced co-release of GABA and glutamate on DG granule cells. a Diagram illustrating the site of the bilateral injections of AAV-DIO-ChR2-EYFP in VGLUT2-cre mice, of light stimulations and of the recorded patch clamp electrode in the DG of the VGLUT2-ChR2-EYFP mice. b Montage illustrating the position within the dentate granule cell layer of the biocytin-filled cells reconstructed after patch clamp recordings and EYFP-labeled terminals originating from SuML neurons. $\mathbf{d}, \mathbf{f}, \mathbf{h}, \mathbf{j}, \mathbf{n}$ Examples of light induced PSCs recorded in DG cells illustrated in $\mathbf{c}, \mathbf{e}, \mathbf{g}, \mathbf{i}, \mathbf{m}$. d Light induced post-synaptic currents (PSCs) recorded at different holding potentials in DG neuron; short black bar above the upper trace shows the time moment and duration of light stimulus. Note at -70 to - $50 \mathrm{mV}$ holdings the PSCs had negative-going direction (inward) currents. They were positive-going (outward) at $-10 \mathrm{mV}$ and $0 \mathrm{mV}$ but displayed both negative- and positive-going phases when the neuron membrane was clamped at $-30 \mathrm{mV}$. This suggests that light stimulation induces two types of postsynaptic currents. Indeed, the application of glutamate receptor blockers NBQX and AP5 (f, red traces) inhibited inward (f, negative going) component of PSCs recorded at negative holding potentials (Vh), but had only small effect on outward component (positive going) recorded at positive $\mathrm{Vh}$. Inversely, the outward component of light induced PSCs was sensitive to GABA A receptor inhibitor gabazine (h, green vs blue traces). This suggests that these PSCs are generated by simultaneous activation of glutamate and GABA post-synaptic receptors. Interestingly at $-50 \mathrm{mV}$ holding, PSC was larger without gabazine (h, blue versus green trace), probably because GABA-mediated current hyperpolarizes the membrane potential increasing thus the driving force for the glutamatergic current. In voltage-clamp condition such an interaction is possible if the pool of postsynaptic GABA Rs is close to the postsynaptic Glu R pool. $\mathbf{k}$, I Effect of glutamate and GABA A receptors blockers on the peak amplitude of light pulse evoked PSC recorded at $-70 \mathrm{mV}$ (red circles) and $10 \mathrm{mV}$ (green circles) holding potentials. For each Vh the peak amplitudes of first 10 PSCs were averaged and used then as a normalization factor for all peak amplitude recorded at given potential. Each point and error bars corresponds the mean \pm SD of PSC normalized amplitude recorded in 5 DG neurons. In regular ACSF the repetitive light pulses $(5 \mathrm{~ms}, 0.05 \mathrm{~Hz})$ evoked PCS of relatively stable amplitude $(1.00 \pm 0.26$, L left-hand side red trace-an example of averaged response of one neuron). The application of glutamate receptor blockers $(10 \mu \mathrm{M}$ NBQX $+40 \mu \mathrm{M}$ D-AP5) reduced the peak amplitude by $84 \%(0.16 \pm 0.15, p<0.01, n=5$, paired Wilcoxon test). The remaining response was seen in 4 from 5 neurons and is probably due to GABA A mediated current because at $-70 \mathrm{mV}$ the driving force for chloride driven currents is close to but not zero (l, red trance in the middle), therefore, in case of GABA massive release some inward current is still possible. Indeed a switch to $\mathrm{Vh}=10 \mathrm{mV}$ revealed a huge PSC response to light stimulation (l, green trace in the middle) whose amplitude was stable $(1.00 \pm 0.20)$ but progressively reduced to $12 \%$ $(0.12 \pm 0.16, p<0.01, n=5$, paired Wilcoxon test $)$ by the addition of $10 \mu \mathrm{M}$ bicuculline to the ACSF already containing GluR blockers (1, green trace at left). In 3 from 5 neurons, 6 min-lasting bicuculline application completely abolished response to light pulses. In two neurons the remaining current is probably due to the competitive character of bicuculline induced inhibition, i.e. in case of high GABA release $10 \mu \mathrm{M}$ of bicuculline may be not sufficient to all receptors inhibition. $\mathbf{m}, \mathbf{n}$ The identical light stimulation (5 ms, 10\%, 50\% 90\% max power LED) of fibers and axon terminals expressing EYFP on slices of control VGLUT2-EYFP mice $(n=3)$ did not evoke any response in the recorded granule cells $(n=5)$ confirming that $\mathrm{ChR} 2$ activation is required to obtain the PSCs. Scale bar $\mathbf{c}, \mathbf{e}, \mathbf{g}, \mathbf{i}, \mathbf{m}, 20 \mu \mathrm{m}$ 
A
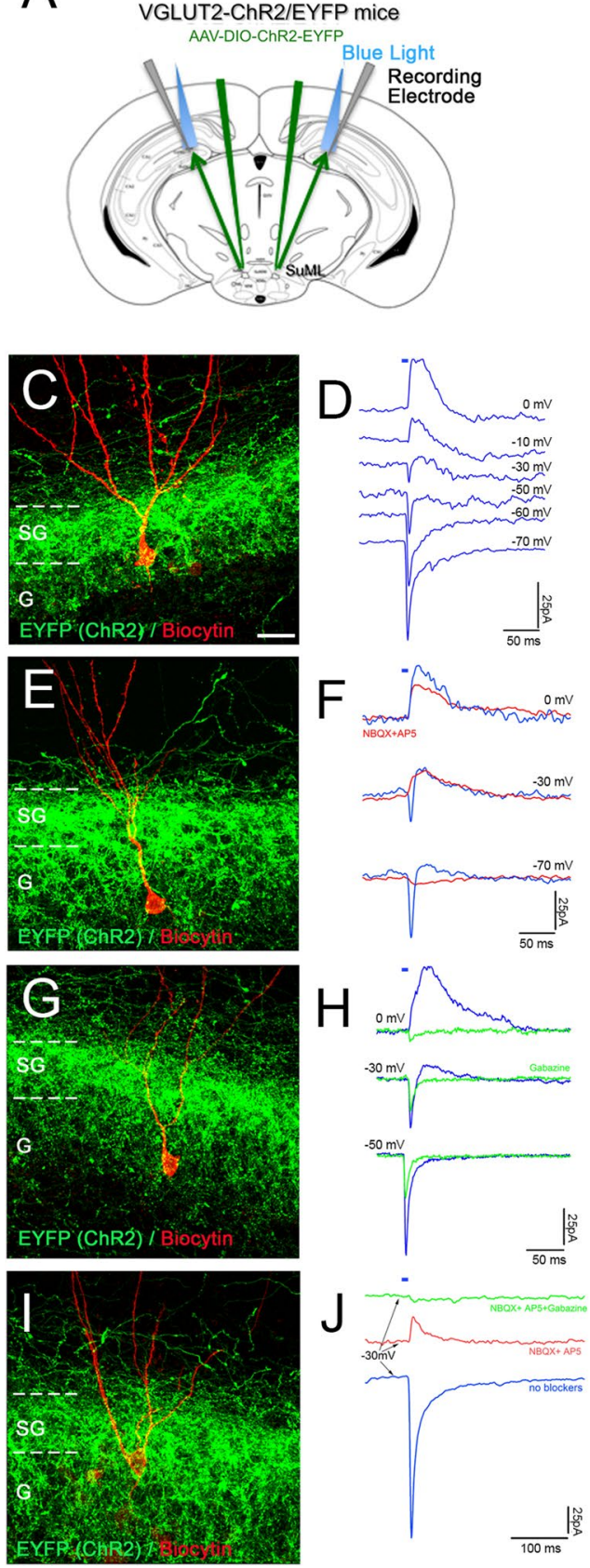

$\mathrm{H}$
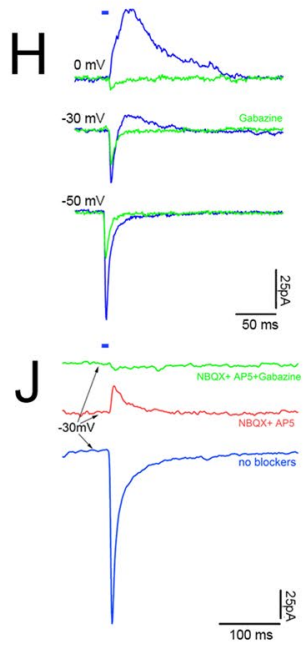
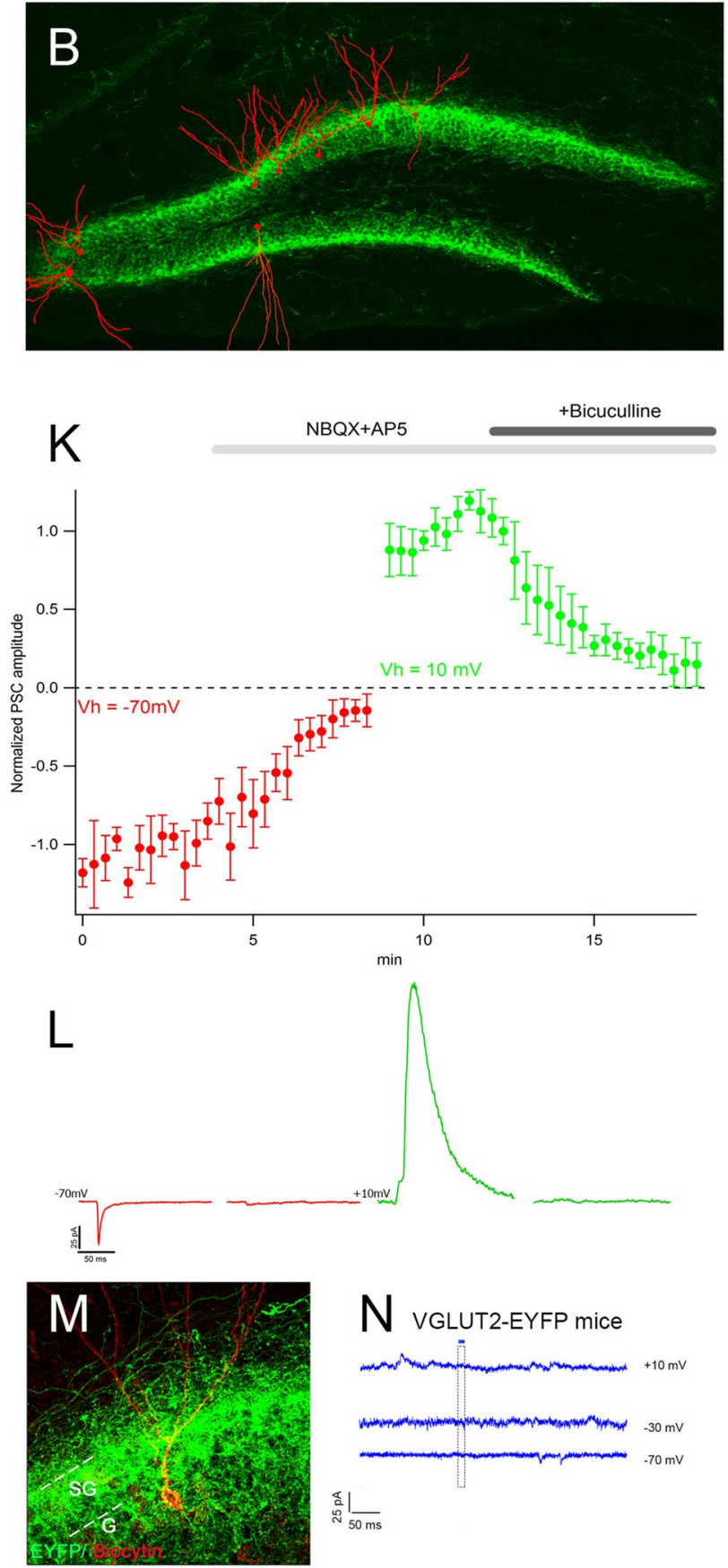

$N$ vglut2-Eyfp mice

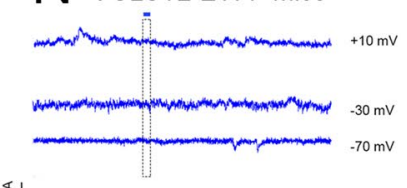

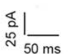


(Fig. 4b). These GCs were additionally recorded at different holding potentials. At $-10 \mathrm{mV}$ and $+10 \mathrm{mV}$ (close to the reversal potential of the glutamatergic receptor-mediated currents), only the outward (positive going) synaptic current was observed (Fig. 4d, see also f, h). From $-70 \mathrm{mV}$ to $-50 \mathrm{mV}$ (close to the reversal potential of GABA-A receptor-mediated currents) only the inward (negative going) synaptic current was observed (Fig. 4d, see also f, h). At intermediate holdings, from -30 to $-20 \mathrm{mV}$, the light-evoked synaptic currents displayed both inward and outward components (Fig. 4d, f, h). Using a pharmacological approach, we confirmed the nature of these currents. Bath application of a mixture of AMPA/ Kainate and NMDA receptor antagonists (NBQX $10 \mu \mathrm{M}$ and D-AP5 $40 \mu \mathrm{M}$ ) abolished the inward component (Fig. 4f, k, 1 , red trace) while GABA-A receptor blockers inhibited the outward component (Fig. 4h, j-gabazine; k, l-bicuculline, green trace). Quantitative analysis performed in 5 DG neurons (Fig. 4k) further illustrated that in regular ACSF the repetitive light pulses $(5 \mathrm{~ms}, 0.05 \mathrm{~Hz}$ ) evoked postsynaptic currents (PSC) of relatively stable amplitude $(1.00 \pm 0.26$ normalized, Fig. 4k, 1, lef- hand side red trace is an example of an averaged response of one neuron). The application of glutamate receptor blockers $(10 \mu \mathrm{M}$ NBQX $+40 \mu \mathrm{M}$ D-AP5) reduced the peak amplitude by $84 \%(0.16 \pm 0.15, p<0.01$, $n=5$, paired Wilcoxon test). The remaining response seen in 4 out of 5 neurons was probably due to GABA-A mediated current because at $-70 \mathrm{mV}$ the driving force for chloride driven currents is close to but is not zero (Fig. 4k, 1, red trace in the middle). Therefore, in response to large amount of GABA being released, some inward current is still possible. Indeed, a switch to $\mathrm{Vh}=10 \mathrm{mV}$ revealed a large PSC response to light stimulation (Fig. 4l, green trace in the middle) which had a stable amplitude $(1.00 \pm 0.20$ normalized $)$ and was subsquently reduced to $12 \%(0.12 \pm 0.16, p<0.01$, $n=5$, paired Wilcoxon test) by the addition of $10 \mu \mathrm{M}$ bicuculline to the ACSF already containing GluR blockers (Fig. 41, green trace at left). In 3 out of 5 neurons, bicuculline completely abolished the synaptic response to light pulses after 6 min of drug perfusion. In two neurons, the small remaining current was probably due to the fact that bicuculline is a competitive antagonist and can be displaced when large quantities of GABA are released. All these results indicate that the inward synaptic current component is mediated by glutamate and the outward component by GABA. Importantly, the disappearance of the inward (glutamatergic) component induced by the light stimulation in the presence of NBQX
Fig. 5 Effects of light stimulation of axonal terminals from SuML neurons innervating the dorsal DG on DG LFP, EEG recordings and behavior of VGLUT2-ChR2-EYFP mice during WK, SWS and PS. a, $\mathbf{e}, \mathbf{i}$ Examples of raw recording for EMG, LFP recorded in the DG and parietal cortex EEG as well as associated time-frequency analysis of LFP and EEG in a VGLUT2-ChR2-EYFP mouse during WK (a), SWS (e) and PS (i). The blue bar represents the optogenetic stimulation period ( $20 \mathrm{~Hz}$ with pulses of $10 \mathrm{~ms}$ for $10 \mathrm{~s}$ ). b, c, f, g, j, k LFP power spectra between 0 and $18 \mathrm{~Hz}$ and 60-100 Hz in VGLUT2ChR2-EYFP mouse before and during light stimulation $(20 \mathrm{~Hz} 10 \mathrm{~s}$, pulses of $10 \mathrm{~ms}$, during $4 \mathrm{~h}$ ) during WK (b, c), SWS (f, g) and PS (j, k). An effect was clearly visible on the LFP in all vigilance states. The stimulation induced a slight increase in the theta power during WK $(\mathbf{a}, \mathbf{b})$ and a major increase in theta power and frequency during PS (i, $\mathbf{j})$ as well as a clear reduction of slow wave oscillation during SWS $(\mathbf{e}, \mathbf{f})$. The stimulation during WK and PS also increased the power of gamma $(\mathbf{c}, \mathbf{k})$. Light stimulation during WK increased locomotor activity reflected by an increase of EMG signal in VGLUT2ChR2-EYFP mice (a, d, blue trace) as compared to control VGLUT2EYFP mice (d, gray trace). h Light stimulation during SWS induced awakening reflected by a significant increase of the awakening percentage after stimulation in most VGLUT2-ChR2-EYFP mice (range $30-100 \%$ ) as compared to that observed in control VGLUT2-EYFP mice (range $0-30 \%$ ). 1 In contrast no significant difference in the percentage of awakening was observed between VGLUT2-CHR2-EYFP and control VGLUT2-EYFP mice when stimulation was performed during PS

and D-AP5 antagonists (red trace) did not affect the outgoing (GABAergic) currents (Fig. 4f). In two GCs (Fig. 4i), the disappearance of the glutamate response (incoming current recorded at $-30 \mathrm{mV}$ ) in the presence of NBQX and AP5 (red trace) unmasked the GABAergic component (outward current) that was abolished after addition of gabazine (green trace) (Fig. 4j).

The very short latency of the glutamatergic inward and GABAergic outward currents recorded in a GC after light stimulation of ChR2 containing axon terminals, and the persistence of GABAergic outflow current in the presence of NBQX and AP5 indicate that the GABAergic current results from direct light-stimulated GABA release and not from the excitation of DG GABAergic neurons by light stimulated glutamate release. Together, these results suggest that activation of SuML axon terminals produce monosynaptic glutamatergic and GABAergic currents on their targets.

Finally, we verified that light stimulation $(5 \mathrm{~ms}, 10 \%$, $50 \% 90 \%$ max power LED) of fibers and axon terminals 


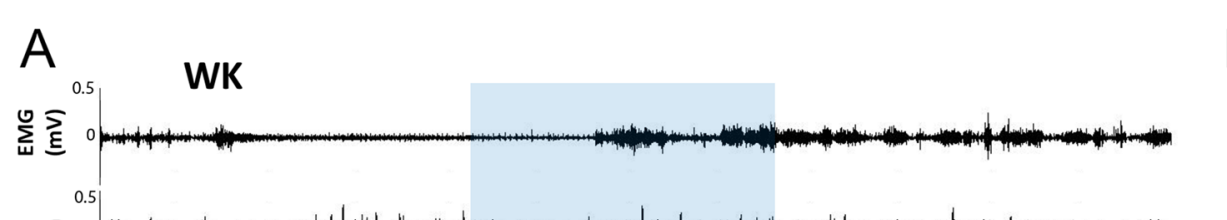

B

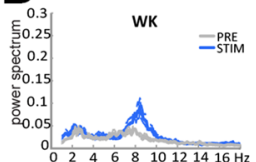

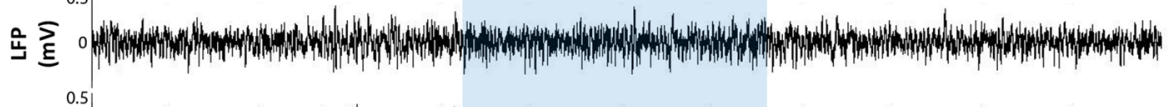

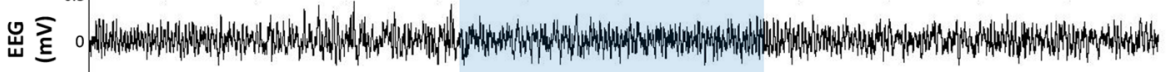

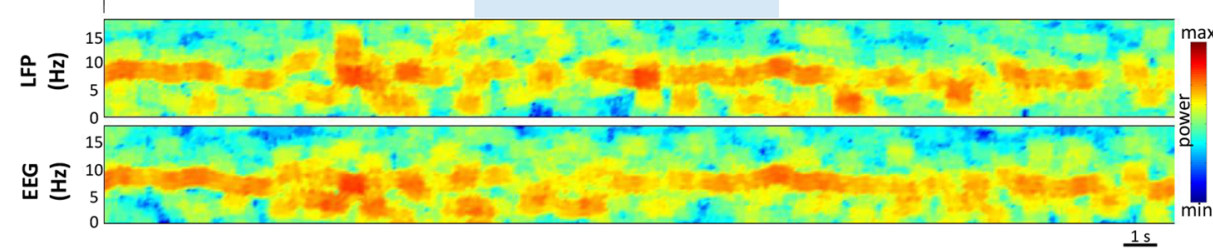

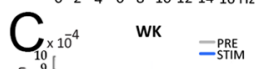

है
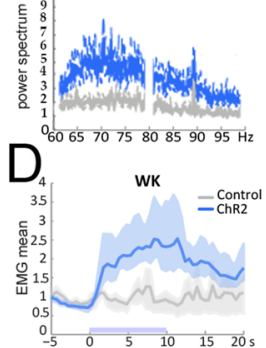

E

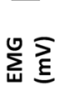

SWS

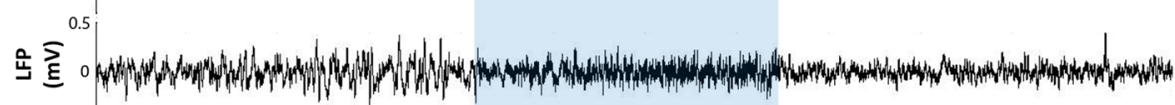

总疋 $\mathcal{S}^{0.5}$.

논 좃

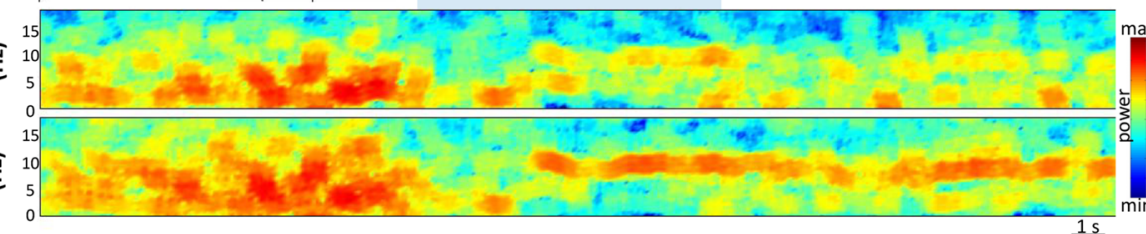

F

SWS

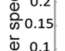

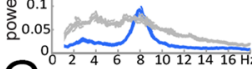

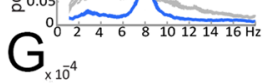

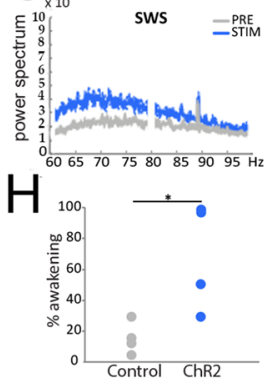

I
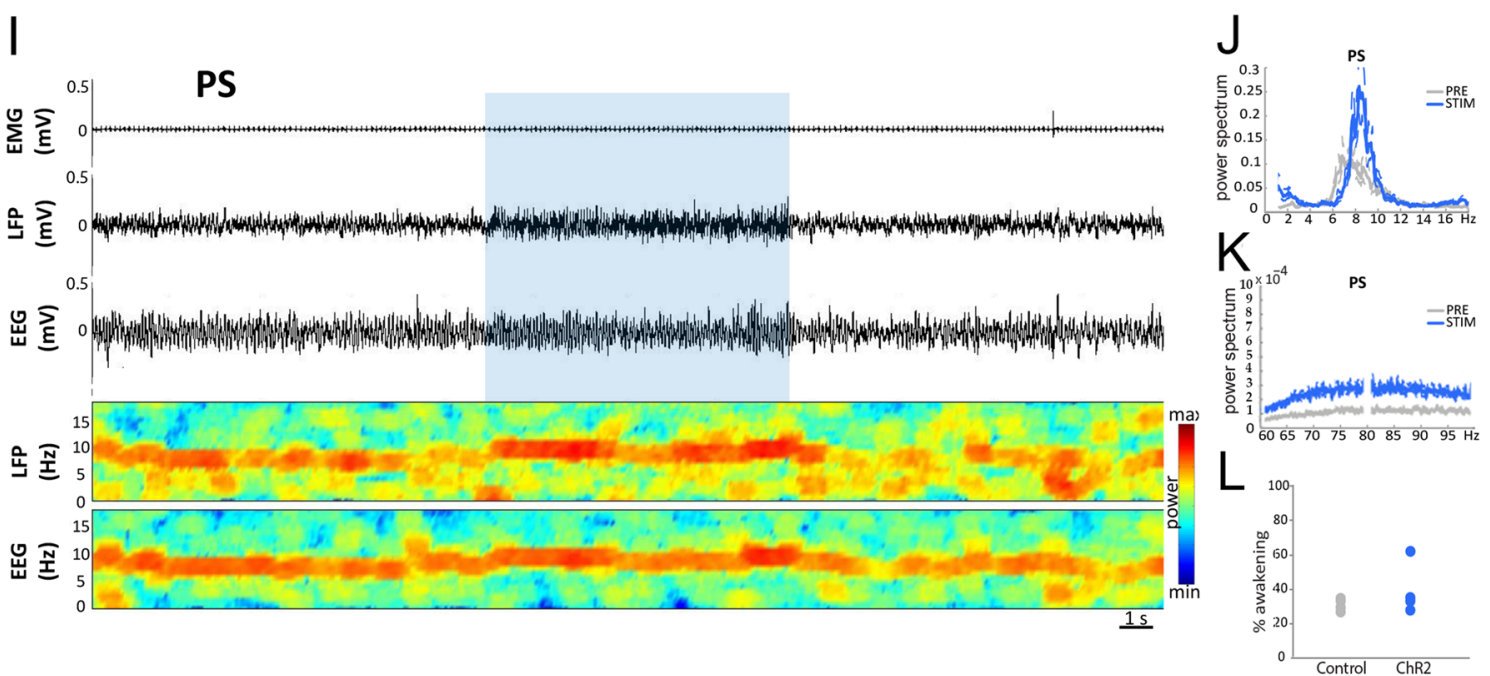

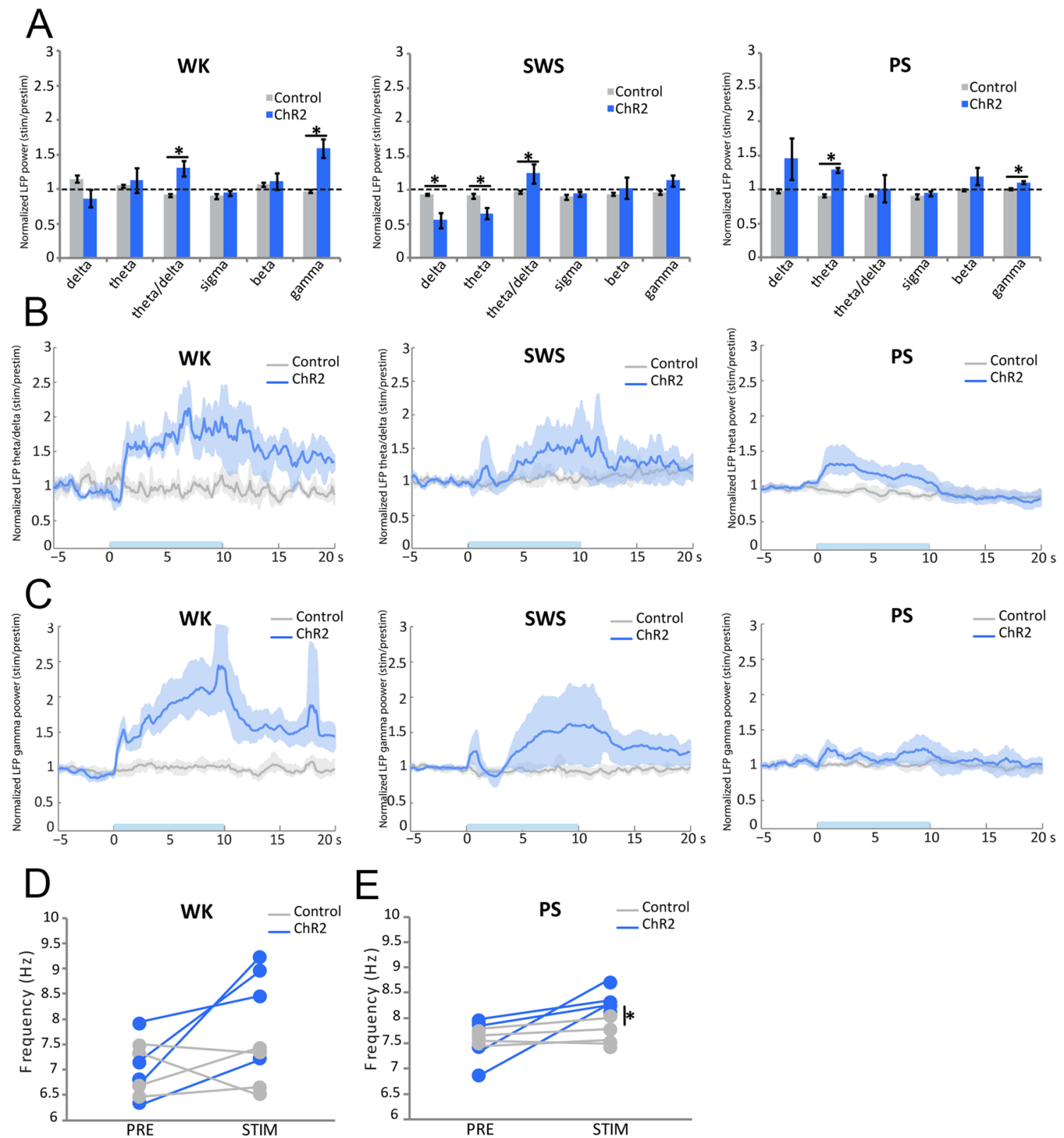

Fig. 6 Comparison of the effects of light stimulation of axonal terminals from SuML neurons innervating the dorsal DG on DG LFP performed during WK, SWS and PS between VGLUT2-ChR2-EYFP and control VGLUT2-ChR2-EYFP mice. a Ratio of power recorded during light stimulation to that recorded before the stimulation $(20 \mathrm{~Hz}, 10 \mathrm{~s}$ for $4 \mathrm{~h}$ with pulses $10 \mathrm{~ms}$ ) for the different frequency bands (delta, theta, gamma) of LFP in VGLUT2-ChR2-EYFP $(n=4)$ and control VGLUT2-EYFP $(n=4)$ mouse groups during WK, SWS and PS. These ratios illustrate the following: an increased power in the theta frequency band during stimulations performed in PS; an increase of theta/delta power during the stimulation when performed in WK and SWS and an increased power in the gamma frequency band when stimulations were performed during WK and PS in the VGLUT2-ChR2-EYFP group. Increased power observed during stimulations observed in the $\mathrm{ChR} 2$ group differed significantly from random variation observed before and after the stimulation in the control group. Significance: Mann Whitney: ${ }^{*} p<0.05$. Theta/ delta (b) and gamma (c) power ratios as a function of time after light stimulation during the different vigilance states. The blue bar on the $x$ axis represents the stimulation period. Shaded regions show $95 \%$ confidence intervals. During WK, a significant increase of theta/ delta (b) and gamma (c) power occurred in VGLUT2-ChR2-EYFP group immediately after the light stimulation. These increases were observed during the entire 20 -s period analyzed. The increase of theta/delta during SWS occurred two to three seconds after the stimulation. The increase of theta (b) and gamma (c) powers during PS occurred immediately after the stimulation and stayed during the 10-s period of stimulation. No difference induced by the stimulation was observed in the control group. d, e Peak frequency analysis of theta before and during optogenetic stimulation in the control group $(n=4)$ and the ChR2 group $(n=4)$. Significance: Mann Whitney, ${ }^{*} p<0.05$ compared to the control group 
expressing EYFP on slices of control VGLUT2-EYFP mice $(n=3)$ did not evoke any response in the recorded GCs $(n=10)$ (Fig. 4m, n).

\section{Effect of optogenetic stimulation of SuML axon terminals innervating the dDG on behavior, LFP and EEG spectral content}

We analyzed the effects of light stimulation of SuML axon terminals innervating the dDG on behavioral states, spectral content of the LFP recorded in dDG and cortical EEG in VGLUT2-ChR2 $(n=4)$ and control VGLUT2-EYFP mice $(n=4)$.

Light activation of SuML axon terminals in VGLUT2ChR2 mice during waking (WK) induced a strong and significant increase in EMG value as compared to control mice (mean EMG ratio, VGLUT2-EYFP $=0.9 \pm 0.03$, VGLUT2-ChR2 $=2.0 \pm 0.31, p=0.0209$ ). The increase in EMG was due to an increase in animal movements (Fig. 5a, d). It was associated with a slight but not significant increase in theta power in the dDG LFP (Figs. 5b, 6a) and the EEG (Fig. 7a) compared to control VGLUT2EYFP mice. However, theta/delta ratio was significantly increased both in the dDG LFP (Fig. 6a, b) and the EEG (Fig. 7c, d) during the stimulation compared to control mice (LFP: VGLUT2-ChR2 $=1.3 \pm 0.11$, VGLUT2-EYFP $=0.9 \pm 0.02, p=0.0209$; EEG: VGLUT2ChR2 $=2.2 \pm 0.31$, VGLUT2-EYFP $=0.9 \pm 0.05$, $p=0.0209)$. The frequency at the theta peak was slightly but not significantly increased during stimulation as compared to control VGLUT2-EYFP animals (LFP: VGLUT2$\mathrm{ChR} 2=8.43 \pm 0.43 \mathrm{~Hz}$, VGLUT2-EYFP $=6.96 \pm 0.23 \mathrm{~Hz}$, $p=0.0833$ ) (Figs. 5b, 6d). Gamma power $(30-100 \mathrm{~Hz}$ ) was significantly increased during the stimulation in VGLUT2-ChR2 mice as compared to control mice in the dDG LFP (VGLUT2-ChR2 $=1.6 \pm 0.13$, VGLUT2$\mathrm{EYFP}=1.0 \pm 0.02, p=0.0209$ ) (Figs. 5c, 6a, c) and in the EEG (VGLUT2-ChR2 $=1.68 \pm 0.05$, VGLUT2$\mathrm{EYFP}=0.98 \pm 0.02, p=0.0209)$ (Fig. 7b, c, e). The increase occurred immediately after the beginning of the stimulation and lasted until the end of it (Figs. 6c, 7e).

Stimulations during SWS induced significantly more often an awakening in VGLUT2-ChR2 than in control VGLUT2-EYFP mice ( $p=0.0209$, VGLUT2-ChR2: $80 \%$ of the cases, range $40-100 \%$; VGLUT2-EYFP: $15 \%$ of the cases, range: $0-30 \%$; Fig. 5e, h). The induced waking state lasted at least the duration of the stimulation and was characterized by a significant increase in theta/ delta ratio (LFP: VGLUT2-ChR2 $=1.3 \pm 0.14$, VGLUT2$\mathrm{EYFP}=1.0 \pm 0.02, p=0.0433$; EEG: VGLUT2$\mathrm{ChR} 2=1.5 \pm 0.27$, VGLUT2-EYFP $=1.0 \pm 0.03$, $p=0.0209$ ) (Figs. 6a, b, 7c, d) and a decrease of delta power both in the dDG LFP (VGLUT2ChR2 $=0.6 \pm 0.11$, VGLUT2-EYFP $=0.9 \pm 0.01$, $p=0.0209$ ) and the EEG (VGLUT2-ChR2 $=0.5 \pm 0.13$, VGLUT2-EYFP $=0.9 \pm 0.02, p=0.0209)$ in VGLUT2ChR2 compared to control VGLUT2-EYFP mice (Figs. 5e, f, 6a, 7a, c). This induced waking state was also associated with an increase in gamma power in the dDG LFP (Figs. 5e, g, 6a, c) and in the EEG (VGLUT2ChR2 $=1.36 \pm 0.16$, VGLUT2-EYFP $=0.99 \pm 0.03$, $p=0.0433$ ) (Fig. 7b, c, e).

In contrast to SWS, light stimulation of SuML axon terminals in the dDG of VGLUT2-ChR2 mice during PS did not induce significantly more awakening in VGLUT2ChR2 mice compared to control VGLUT2-EYFP animals ( $p=0.3865$; Fig. 51).

Light activation of SuML axon terminals during PS in VGLUT2-ChR2 induced a significant increase in theta power compared to VGLUT2-EYFP mice in the dDG LFP (Figs. 5i, j, 6a, b; VGLUT2-ChR $2=1.3 \pm 0.04$, VGLUT2-EYFP $=0.9 \pm 0.02, p=0.209)$ but not in the EEG (Fig. 7c, d; $p=0.773$ ). The frequency at the theta peak was also significantly increased in the dDG LFP compared to control VGLUT2-EYFP animals (VGLUT2$\mathrm{ChR} 2=8.3 \pm 0.13 \mathrm{~Hz}$, VGLUT2-EYFP $=7.7 \pm 0.15 \mathrm{~Hz}$, $p=0.0209$; Fig. 6e). In addition, gamma was significantly increased both in the dDG LFP and the EEG during the stimulation in VGLUT2-ChR2 mice compared to control VGLUT2-EYFP mice (LFP: VGLUT2ChR2 $=1.1 \pm 0.02$, VGLUT2-EYFP $=1.0 \pm 0.01$, $p=0.0209$; EEG: VGLUT2-ChR2 $=1.68 \pm 0.05$, VGLUT2-EYFP $=0.98 \pm 0.02, p=0.0209$; Figs. 5k, 6a, c, 7b, c, e).

\section{Effect of optogenetic activation of SuML axon terminals innervating dDG on cFos expression}

Mouse brains were processed for immunohistochemical detection of cFos in order to assess the effect of SuML 
A

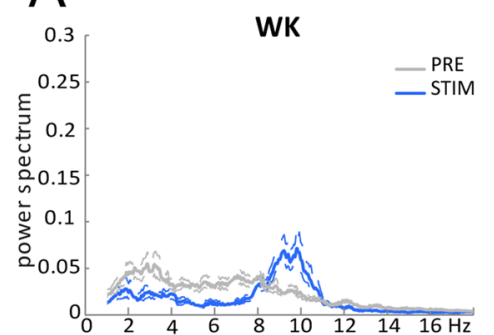

B

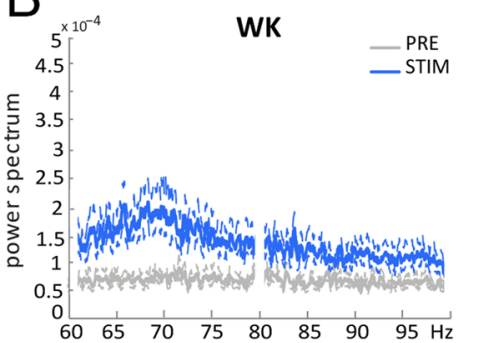

C

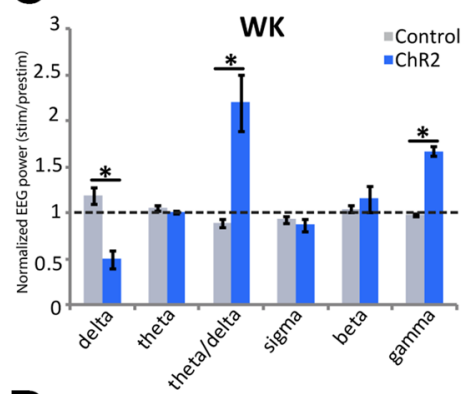

D

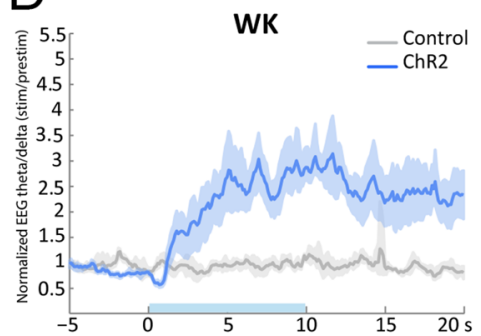

E

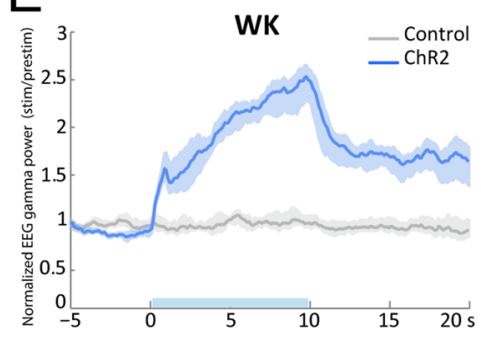

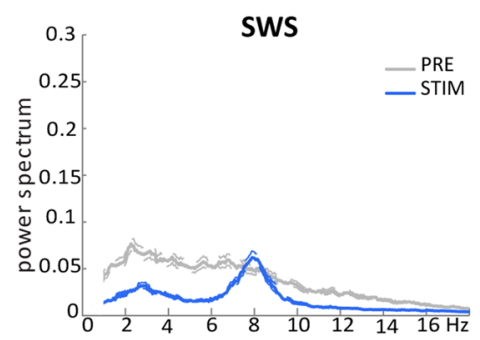
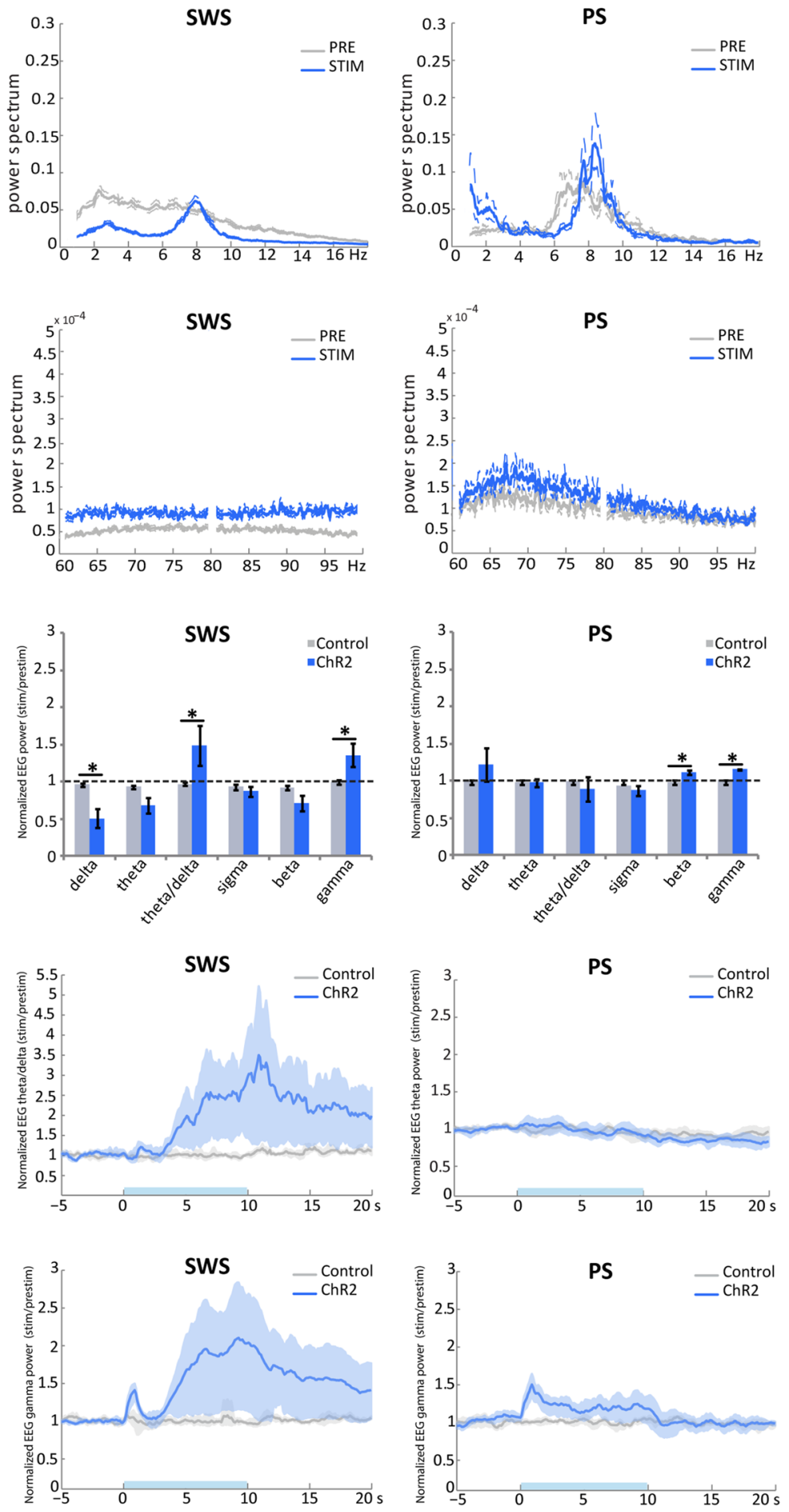
4Fig. 7 Comparison of the effects of light stimulation of axonal terminals from SuML neurons innervating the dorsal DG on EEG performed during WK, SWS and PS between VGLUT2-ChR2-EYFP and control VGLUT2-EYFP mice. a, b EEG power spectra between 0 and $18 \mathrm{~Hz}$ and $60-100 \mathrm{~Hz}$ in a VGLUT2-ChR2-EYFP mouse before and during light stimulation ( $20 \mathrm{~Hz} 10 \mathrm{~s}$, pulses of $10 \mathrm{~ms}$, during $4 \mathrm{~h}$ ) during WK, SWS and PS. An effect was clearly visible on the EEG in all vigilance states. $\mathbf{c}$ Ratio of power recorded during light stimulation to that recorded before the stimulation for the different frequency bands (delta, theta, gamma) of EEG in VGLUT2-ChR2-EYFP $(n=4)$ and control VGLUT2-EYFP $(n=4)$. These ratios illustrate an increase of theta/delta power during the stimulation when performed in WK and SWS and an increased power in the gamma frequency band when stimulations were performed during all vigilance states in the VGLUT2-ChR2-EYFP group. These increased powers during stimulations observed in ChR2 group differed significantly from random variation observed before and after the stimulation in the control group. Significance: Mann Whitney: ${ }^{*} p<0.05$. Theta/delta (d) and gamma (e) power ratios as a function of time after light stimulation during the different vigilance states. The blue bar on the $x$ axis represents the stimulation period. Shaded regions show $95 \%$ confidence intervals. During WK, a significant increase of theta/delta (b) and gamma (c) powers occurred in VGLUT2-ChR2-EYFP group immediately after the light stimulation. These increases were observed during the entire 20 -s period analyzed. The increase of theta/delta during SWS occurred 2- to 3 seconds after the stimulation. The increase of gamma (c) powers during PS occurred immediately after the stimulation and lasted during the 10-s period of stimulation. No increase in theta was observed during PS (c, d) in the EEG. No difference induced by the stimulation was observed in the control group axon terminal activation on DG cell activity. Whereas only some neurons were labeled for cFos in the GCL of the DG in control VGLUT2-EYFP mice $(n=4)$ (Fig. 8a, b), numerous neurons labeled for cFos were observed in the granule cell layer of the dDG in VGLUT2-ChR2 mice $(n=4)$ (Fig. 8c, d). In addition, most of these neurons in the GCL were highly labeled for cFos as compared to those observed in control mice (Fig. 8 compare D with B). Quantitative analysis showed a $241 \%$ increase in the number of labeled cFos neurons in the dDG ipsilateral to the stimulation site $(270 \pm 33)$ as compared to control mice (ipsi: $79 \pm 23 ; p=0.026$; Fig. 8e), whereas no significant difference was observed in the contralateral dDG (VGLUT2ChR2: $178 \pm 21$; VGLUT2-EYFP contra: $90 \pm 33 ; p=0.2$; Fig. 8e). 


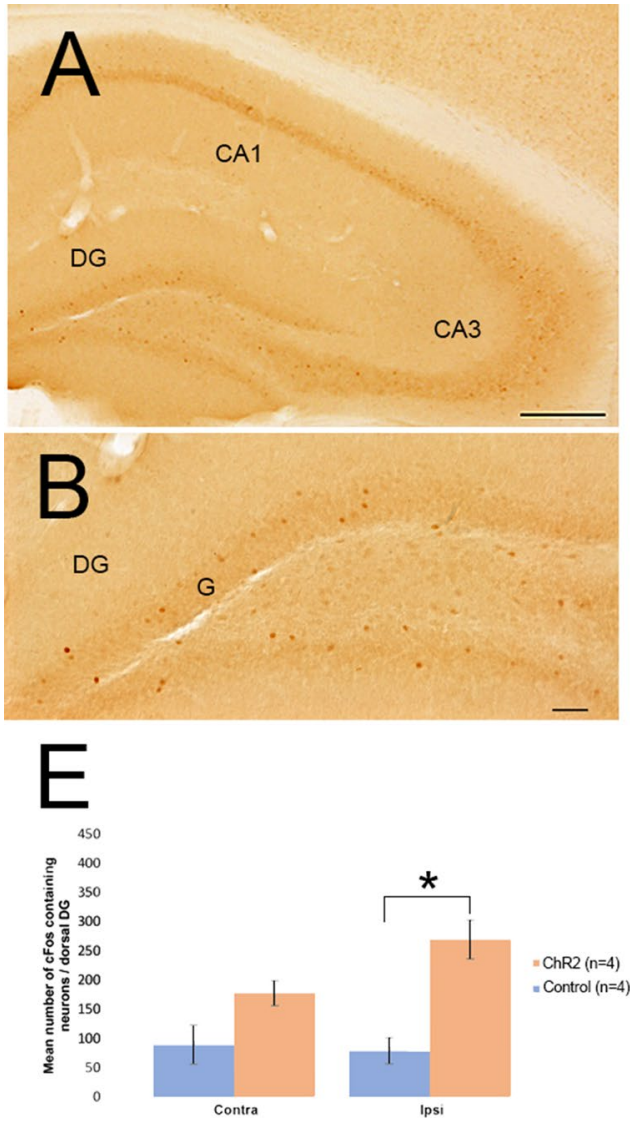

Fig. 8 Activation of DG cells after light stimulation of axon terminals from SuML neurons innervating the DG in VGLUT2-ChR2-EYFP and VGLUT2-EYFP as reflected by cFos immunohistochemical labeling. a-d Immunohistochemical labeling for $\mathrm{cFos}$ on sections of control VGLUT-EYFP $(\mathbf{a}, \mathbf{b})$ and VGLUT2-ChR2-EYFP $(\mathbf{c}, \mathbf{d})$ mice. In control mice $(\mathbf{a}, \mathbf{b})$ some neurons labeled for cFos were observed in the hippocampus. They were scattered in all layers of the CA1, CA3 regions of the hippocampus and the DG. In addition, most of these neurons were lightly labeled for cFos including those observed in the granule cell layer $(\mathrm{G})$ of the DG. In contrast, in VGLUT2-ChR2-

\section{Discussion}

\section{Neuroanatomical and "in vitro" experiments showing the dual glutamate-GABA feature of the SuML-DG pathway}

Our results first establish at the neuroanatomical level that in mice as in rats (Soussi et al. 2010), all SuM neurons innervating the dDG display a dual GABAergic and glutamatergic neurotransmitter phenotype. We further demonstrate that these neurons correspond to a population of SuM cells located just dorsal to the mammillary tract that likely correspond to grandicellular neurons described in rat within the SuML region (Paxinos and Watson 1998) or SuMg (Pan and McNaughton 2004). In addition, our
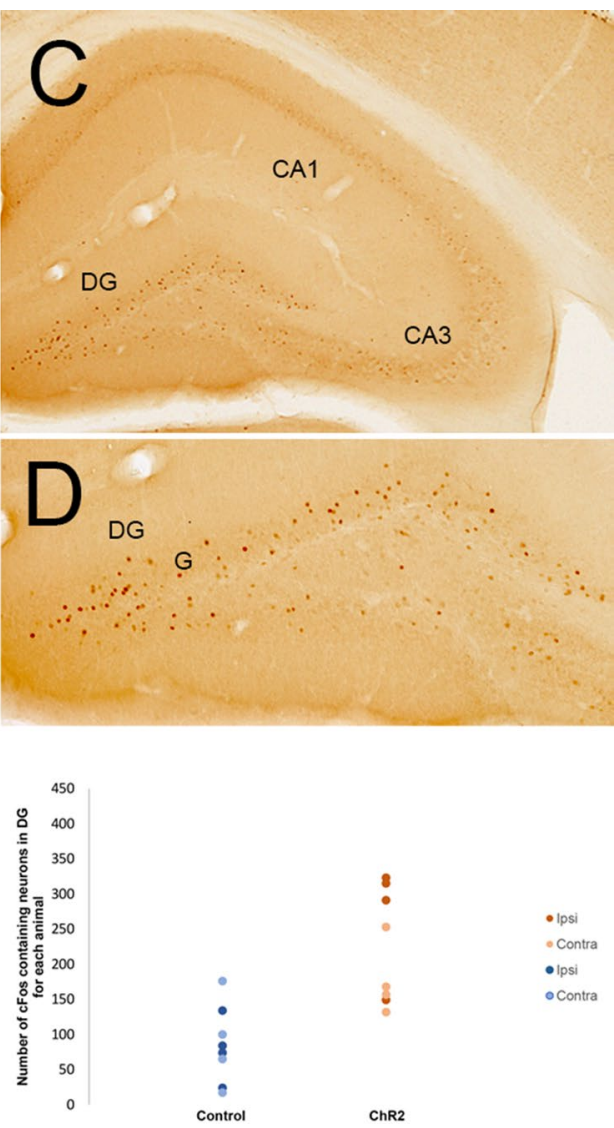

EYFP mice, many c-Fos containing neurons were located in the granule cells layer $(\mathrm{G})$ of the DG, whereas only a few were observed within the CA3 and CA1 regions of the hippocampus. In the granule cell layer, most of these neurons were highly labeled for cFos (d) as compared to those observed in control mice (b). e Quantitative analysis showing that the number of cFos-containing neurons was increased in the granule cell layer of the stimulated DG (ipsi) but not in the contralateral DG (contra) in VGLUT2-ChR2-EYFP mice $(n=4)$ as compared to control mice $(n=4)$. Wilcoxon Rank Sum test $* p<0.05 \%$; scale bars a, c $250 \mu \mathrm{m}$; b, d $50 \mu \mathrm{m}$

EM data show that SuML terminals form asymmetric (presumed excitatory glutamate) synapses onto some GCs and symmetric (presumed inhibitory GABA) synapses onto others as previously described in rat (Boulland et al. 2009; Soussi et al. 2010) and mouse (Root et al. 2018). We further found in this study that one axon bouton from a SuML neuron can form an asymmetric synapse on a GC and a symmetric synapse on another GC. In agreement with these neuroanatomical results, our in vitro electrophysiological experiments show that optical stimulation of SuM axon terminals innervating the dDG induces cotransmission of GABA and glutamate on almost all dentate GCs in line with two recent studies (Pedersen et al. 2017; Hashimotodani et al. 2018). Hashimotodani et al. (2018) further showed that such co-transmission of GABA and 
glutamate induces net excitatory effects on GCs and potentiates GC firing when temporally associated with perforant path inputs. In line with such a hypothesis, after in vivo optic stimulation of SuML axon terminals innervating the dDG in VGLUT2-ChR2 mice, we found that a significant number,, but not all GC neurons below the optic fiber were labeled with cFos. Therefore, these cFos labeled neurons could constitute a population of GCs that are simultaneously activated by the optic stimulation of SuML axon terminals and perforant path inputs.

\section{Effect of optogenetic stimulation of SuML-DG fibers on vigilance states}

Activation of SuML axon terminals projecting to the DG induces an awakening effect in mice when performed during SWS, but not during PS. It has been shown by Renouard et al. (2015) that the SuML-DG pathway is active during PS and, therefore, its stimulation during this state might not induce WK because the pathway is already engaged and its overactivation might, therefore, not to be sufficient to awaken the animal. In contrast, when the stimulation occurs during SWS, an awakening is induced likely because the path is normally inactive during this state. It can be proposed that the stimulation of the DG granule cells by the SuML induces the reactivation of memories and subsequently of structures involved in the exploration leading to an awakening of the animal during SWS. It might also be due to the fact that there is no muscle atonia during this state compared to PS. In line with the latter hypothesis, stimulation during WK induces increased motor and exploratory activity. Such result is in line with the literature since an increase in exploratory activity was reported when stimulating with blue light dDG neurons expressing ChR2 (Kheirbek et al., 2013).

\section{Optogenetic stimulation of SuML-DG fibers increases gamma and theta}

Our study further demonstrates that activation of SuML axon terminals innervating the DG during PS increases theta power and frequency as well as gamma power in the DG LFP and to a minor extent in the EEG. Activation of the SuML fibers during WK also induces an increase of gamma power in the DG LFP and EEG associated with an increased locomotion. During SWS, the activation of the SuML-dDG pathway induced awakening and a switch from delta to theta activity and an increase in gamma power both in DG LFP and EEG.

It has been previously shown that the SuM can exert significant modulatory control of the theta rhythm (Vertes and Kocsis 1997). A large percentage of SuM neurons discharge rhythmically, in phase with theta (Kirk and
McNaughton 1991; Kocsis and Vertes 1994) and this activity is independent of that occurring in the hippocampus. Indeed, neurons in the SuM continue to fire bursts in the theta range frequency after lesion or pharmacological inactivation of the medial septum (Kirk and McNaughton 1991) known to abolish theta rhythmic activity in the hippocampus. Further, electrical stimulation or carbachol injections in the SuM synchronously drive theta phase-locked cells in both septum (Bland et al. 1994) and hippocampus (Colom et al. 1987). In addition, the SuM controls the frequency and amplitude of theta (Kirk and McNaughton 1993). These results indicated that the SuM plays a role in theta occurrence but experiments were performed in anesthetized animals and did not specifically study the SuML-DG pathway like in the present study. Some SuM neurons are known to innervate both the DG and the medial septum (Vertes and McKenna 2000). Therefore, one could argue that the effects that we observe by optic stimulation of axon terminals from SuML neurons innervating the DG could partially reflect the influence of the septum via antidromic activation of these SuML neurons. Although we cannot totally dismiss this contribution, this alone cannot account for the effects observed. In fact, the SuM neurons that project both to the DG and the septum represent less than $10 \%$ of the SuM-DG Neurons (Vertes and McKenna 2000). In addition, the antidromic optical activation is strongly affected by the distance (Tye et al. 2011) and requires high light intensity ( $>30 \mathrm{~mW}$; Ciocchi et al. 2015), whereas we stimulated at $8 \mathrm{~mW}$.

Our results also show that the frequency at the peak of theta is higher during PS than in WK in basal conditions. Further, we found that optical stimulation of SuML-DG fibers during PS induced a similar increase of the frequency at the peak of theta. Besides, lesion of the SuM induced a decrease of theta power specifically during PS (Renouard et al. 2015). On the other hand, lesion of all neurons or specific inactivation of the GABAergic neurons of the medial septum strongly decreases theta during PS and WK (Mitchell et al. 1982; Green and Arduini 1954; Boyce et al. 2016). Our data also show that stimulation of the SuML-DG fibers induces an increase in gamma power in the dDG and also in the EEG both during PS and WK. Interestingly, Montgomery et al. (2008) found by coherence analysis that dentate/CA3 theta and gamma power and synchrony were significantly higher during PS compared with active WK and that, in contrast, gamma power in CA1 and CA3-CA1 gamma coherence showed significant decreases during PS. These and our results strongly suggest that the medial septum GABAergic neurons induce theta both during WK and PS, whereas the increase of theta and gamma power and theta frequency occurring in the DG during PS compared to WK is induced by the projection from the SuML. 


\section{Functional role of the SumL-DG pathway: a hypothesis}

Earlier studies have suggested a key role of dentate GCs in emotional learning and memory (Schwegler et al. 1981) and complex spatial learning (Bernasconi-Guastalla et al. 1994). More recently, it has been shown that GCs of the DG are instrumental in the formation of the contextual component of fear memories (Denny et al. 2014) and spatial discrimination (McHugh et al. 2007). In particular, it has been shown that small populations of GCs (2-4\%) representing memory engrams are specifically activated when the animals are exposed to a specific context. These cells are reactivated each time the animal is re-exposed to the same context (Schmidt et al. 2012). Optogenetic activation in a different context of a DG engram activated during contextual fear conditioning induces freezing (Liu et al. 2012). Conversely, inactivation during contextual fear memory recall of the DG GCs activated during encoding decreases freezing (Denny et al. 2014). These results indicate that activation of memory engrams composed of DG GCs cells is necessary for spatial learning. Interestingly, in these studies, the mean number of neurons labeled with cFos or Arc in one section $(35 \mu \mathrm{m})$ of the DG after contextual fear conditioning was in the 30-60 range. In the present study, during stimulation of the SuML fibers in the DG, a mean of 67 DG cells were expressing cFos in the DG per section $(40 \mu \mathrm{m})$. Finally, Renouard et al. (2015) counted a mean of 68 Arc- and 66 cFos-labeled neurons in one DG section after PS hypersomnia in rats. Therefore, approximatively the same number of DG GCs cells is activated during encoding of a contextual fear memory, stimulation of the SuML fibers in the DG and PS hypersomnia. Even if quite speculative, first, the fact that a similar number of cells is activated when stimulating SuML terminals as during PS hypersomnia suggests that the activation seen during PS is likely due to activation of the SuML-DG pathway. Second, the fact that a similar number of cells are activated during a memory task as during PS hypersomnia and stimulation of the SuML fibers suggests that memory engrams could be activated in these two conditions. Therefore, the induction of an active behavior when the stimulation is made during WK could be due to the activation of memory engrams. Since only a limited number of GCs cells are activated during PS and SuML terminals stimulation despite the fact that a large number of GC neurons seems to be innervated by SuML axon terminals, it also suggests that activation of these cells may be due to a conjunction of the SuML input with another excitatory input. It can be proposed that the medial entorhinal is involved since it provides the main excitatory afferent to the DG involved in its activation during memory encoding (Sasaki et al. 2015) and it is one of the few limbic structures activated during PS (Renouard et al. 2015). Such hypothesis remains to be tested using optogenetic manipulation of the activated neurons during PS.

Acknowledgements We thank the animal facility (CEFOS, AMU, Marseille), the imaging facility (INPHIM, AMU, Marseille) and the electron microscope facility (IBDM, Marseille).

Author contributions Experiments and data analysis were performed by FB, LC, HE, AII, JS. AG and HB contributed to experiments. JC and P-AL contributed to development of analytic tools. NEA contributed to data analysis. FB, contributed to first draft of the manuscript. EKM, IS and CB contributed to the study design and writing review. P-HL and ME designed and supervised the study and wrote the manuscript. All authors have read and approved the final version of the manuscipt.

Funding This work was supported by INSERM and Aix-Marseille University (M. E., H. E., C. B., L. C., AI. I., A. G., J. S.); Partenariats Hubert Curien (PHC) IMHOTEP (M. E., H. E., NE. A.); Agence Universitaire Francophone (H. E.); Ministry of Scientific Research, Egypt (H. E., NE. A.); CNRS, Fondation pour la recherche médicale (FRM), Société Française de Recherche et Médecine du Sommeil (SFRMS), University Claude Bernard of Lyon (F. B., P-H. L., P-A. L., S. A.) and NS104590 (I. S., E. K-M.).

\section{Compliance with ethical standards}

Conflict of interest The authors declare that they have no conflict of interest.

Research involving animals and ethical approval All animal experiments were performed according to the National Institutes of Health guidelines and the European communities Council Directive of 86/609/ EEC and were approved by the University of Aix-Marseille and Lyon University Chancellor's Animal Research Committees. This article does not contain any studies with human participants performed by any of the authors.

Open Access This article is licensed under a Creative Commons Attribution 4.0 International License, which permits use, sharing, adaptation, distribution and reproduction in any medium or format, as long as you give appropriate credit to the original author(s) and the source, provide a link to the Creative Commons licence, and indicate if changes were made. The images or other third party material in this article are included in the article's Creative Commons licence, unless indicated otherwise in a credit line to the material. If material is not included in the article's Creative Commons licence and your intended use is not permitted by statutory regulation or exceeds the permitted use, you will need to obtain permission directly from the copyright holder. To view a copy of this licence, visit http://creativecommons.org/licenses/by/4.0/.

\section{References}

Bernasconi-Guastalla S, Wolfer DP, Lipp H-P (1994) Hippocampal mossy fibers and swimming navigation in mice: correlations with size and left-right asymmetries. Hippocampus 4:53-64

Billwiller F, Renouard L, Clement O, Fort P, Luppi PH (2017) Differential origin of the activation of dorsal and ventral dentate gyrus granule cells during paradoxical (REM) sleep in the rat. Brain Struct Funct 222:1495-1507 
Bland BH, Oddie SD, Colom LV, Vertes RP (1994) Extrinsic modulation of medial septal cell discharges by the ascending brainstem hippocampal synchronizing pathway. Hippocampus 4:649-660

Bolte S, Cordelières FP (2006) A guided tour into subcellular colocalization analysis in light microscopy. J Microsc 224:213-232

Borhegyi Z, Leranth C (1997) Distinct substance P- and calretinincontaining projections from the supramammillary area to the hippocampus in rats; a species difference between rats and monkeys. Exp Brain Res 115:369-374

Boulland JL, Jenstad M, Boekel AJ, Wouterlood FG, Edwards RH, Storm-Mathisen J, Chaudhry FA (2009) Vesicular glutamate and GABA transporters sort to distinct sets of vesicles in a population of presynaptic terminals. Cereb Cortex 19:241-248

Boyce R, Glasgow SD, Williams S, Adamantidis A (2016) Causal evidence for the role of REM sleep theta rhythm in contextual memory consolidation. Science 352:812-816

Bras H, Gaytán SP, Portalier P, Zanella S, Pásaro R, Coulon P, Hilaire G (2008) Prenatal activation of 5-HT 2A receptor induces expression of 5-HT 1B receptor in phrenic motoneurons and alters the organization of their premotor network in newborn mice. Eur J Neurosci 28:1097-1107

Ciocchi S, Passecker J, Malagon-Vina H, Mikus N, Klausberger $\mathrm{T}$ (2015) Brain computation. Selective information routing by ventral hippocampal CA1 projection neurons. Science 348:560-563

Colom LV, Ford RD, Bland BH (1987) Hippocampal formation neurons code the level of activation of the cholinergic septohippocampal pathway. Brain Res 410:12-20

Coulon P, Bras H, Vinay L (2011) Characterization of last-order premotor interneurons by transneuronal tracing with rabies virus in the neonatal mouse spinal cord. J Comp Neurol 519:3470-3487

Denny CA, Kheirbek MA, Alba EL, Tanaka KF, Brachman RA, Laughman KB, Tomm NK, Turi GF, Losonczy A, Hen R (2014) Hippocampal memory traces are differentially modulated by experience, time, and adult neurogenesis. Neuron 83:189-201

Gonzalo-Ruiz A, Alonso A, Sanz JM, Llinás RR (1992) Afferent projections to the mammillary complex of the rat, with special reference to those from surrounding hypothalamic regions. J Comp Neurol 321:277-299

Green JD, Arduini AA (1954) Hippocampal electrical activity in arousal. J Neurophysiol 17:533-557

Haglund L, Swanson LW, Köhler C (1984) The projection of the supramammillary nucleus to the hippocampal formation: an immunohistochemical and anterograde transport study with the lectin PHA-L in the rat. J Comp Neurol 229:171-185

Hashimotodani Y, Karube F, Yanagawa Y, Fujiyama F, Kano M (2018) Supramammillary nucleus afferents to the dentate gyrus co-release glutamate and GABA and potentiate granule cell output. Cell Rep 25:2704-2715

Kheirbek MA, Drew LJ, Burghardt NS, Costantini DO, Tannenholz L, Ahmari SE, Zeng H, Fenton AA, Hen R (2013) Differential control of learning and anxiety along the dorsoventral axis of the dentate gyrus. Neuron 77:955-968

Kirk IJ, McNaughton N (1991) Supramammillary cell firing and hippocampal rhythmical slow activity. NeuroReport 2:723-725

Kirk IJ, McNaughton N (1993) Mapping the differential effects of procaine on frequency and amplitude of reticularly elicited hippocampal rhythmical slow activity. Hippocampus 3:517-525

Kocsis B, Kaminski M (2006) Dynamic changes in the direction of the theta rhythmic drive between supramammillary nucleus and the septohippocampal system. Hippocampus 16:531-540

Kocsis B, Vertes RP (1994) Characterization of neurons of the supramammillary nucleus and mammillary body that discharge rhythmically with the hippocampal theta rhythm in the rat. $\mathbf{J}$ Neurosci 14:7040-7052
Kocsis K, Kiss J, Csáki A, Halász B (2003) Location of putative glutamatergic neurons projecting to the medial preoptic area of the rat hypothalamus. Brain Res Bull 61:459-468

Leranth C, Kiss J (1996) A population of supramammillary area calretinin neurons terminating on medial septal area cholinergic and lateral septal area calbindin-containing cells are aspartate/ glutamatergic. J Neurosci 16:7699-7710

Liu X, Ramirez S, Pang PT, Puryear CB, Govindarajan A, Deisseroth K, Tonegawa $S$ (2012) Optogenetic stimulation of a hippocampal engram activates fear memory recall. Nature 484:381-385

Maglóczky Z, Acsády L, Freund TF (1994) Principal cells are the postsynaptic targets of supramammillary afferents in the hippocampus of the rat. Hippocampus 4:322-334

Maquet P, Laureys S, Peigneux P, Fuchs S, Petiau C, Phillips C, Aerts J, Del Fiore G, Degueldre C, Meulemans T, Luxen A, Franck G, Van Der Linden M, Smith C, Cleeremans A (2000) Experiencedependent changes in cerebral activation during human REM sleep. Nat Neurosci 3:831-836

McHugh TJ, Jones MW, Quinn JJ, Balthasar N, Coppari R, Elmquist JK, Lowell BB, Fanselow MS, Wilson MA, Tonegawa S (2007) Dentate gyrus NMDA receptors mediate rapid pattern separation in the hippocampal network. Science 317:94-99

Mitchell SJ, Rawlins JN, Steward O, Olton DS (1982) Medial septal area lesions disrupt theta rhythm and cholinergic staining in medial entorhinal cortex and produce impaired radial arm maze behavior in rats. J Neurosci 2:292-302

Montgomery SM, Sirota A, Buzsáki G (2008) Theta and gamma coordination of hippocampal networks during waking and rapid eye movement sleep. J Neurosci 28:6731-6741

Nitsch R, Leranth C (1996) GABAergic neurons in the rat dentate gyrus are innervated by subcortical calretinin-containing afferents. J Comp Neurol 364:425-438

Pan WX, McNaughton N (2002) The role of the medial supramammillary nucleus in the control of hippocampal theta activity and behaviour in rats. Eur J Neurosci 16:1797-1809

Pan WX, McNaughton N (2004) The supramammillary area: its organization, functions and relationship to the hippocampus. Prog Neurobiol 74:127-166

Pasquier DA, Reinoso-Suarez F (1976) Direct projections from hypothalamus to hippocampus in the rat demonstrated by retrograde transport of horseradish peroxidase. Brain Res 108:165-169

Pasquier DA, Reinoso-Suarez F (1978) The topographic organization of hypothalamic and brain stem projections to the hippocampus. Brain Res Bull 3:373-389

Paxinos G, Franklin KB (2005) The mouse brain in stereotaxic coordinates, 5th edn. Academic Press, San Diego

Paxinos G, Watson C (1998) The rat brain in stereotaxic coordinates. Academic Press, Sydney

Pedersen NP, Ferrari L, Venner A, Wang JL, Abbott SBG, Vujovic N, Arrigoni E, Saper CB, Fuller PM (2017) Supramammillary glutamate neurons are a key node of the arousal system. Nat Commun 8:1405

Persson S, Boulland JL, Aspling M, Larsson M, Fremeau RT Jr, Edwards RH, Storm-Mathisen J, Chaudhry FA, Broman J (2006) Distribution of vesicular glutamate transporters 1 and 2 in the rat spinal cord, with a note on the spinocervical tract. J Comp Neurol 497:683-701

Raux H, Iseni F, Lafay F, Blondel D (1997) Mapping of monoclonal antibody epitopes of the rabies virus P protein. J Gen Virol 78:119-124

Renouard L, Billwiller F, Ogawa K, Clément O, Camargo N, Abdelkarim M, Gay N, Scoté-Blachon C, Touré R, Libourel PA, Ravassard P, Salvert D, Peyron C, Claustrat B, Léger L, Salin P, Malleret G, Fort P, Luppi PH (2015) The supramammillary nucleus and 
the claustrum activate the cortex during REM sleep. Sci Adv 1:e1400177

Richmond MA, Yee BK, Pouzet B, Veenman L, Rawlins JN, Feldon J, Bannerman DM (1999) Dissociating context and space within the hippocampus: effects of complete, dorsal, and ventral excitotoxic hippocampal lesions on conditioned freezing and spatial learning. Behav Neurosci 113:1189-1203

Root DH, Zhang S, Barker DJ, Miranda-Barrientos J, Liu B, Wang HL, Morales M (2018) Selective brain distribution and distinctive synaptic architecture of dual glutamatergic-GABAergic neurons. Cell Rep 23:3465-3479

Santín LJ, Aguirre JA, Rubio S, Begega A, Miranda R, Arias JL (2003) c-Fos expression in supramammillary and medial mammillary nuclei following spatial reference and working memory tasks. Physiol Behav 78:733-739

Saper CB (1985) Organization of cerebral cortical afferent systems in the rat. II. Hypothalamocortical projections. J Comp Neurol 237:21-46

Sapin E, Lapray D, Bérod A, Goutagny R, Léger L, Ravassard P, Clément O, Hanriot L, Fort P, Luppi PH (2009) Localization of the brainstem GABAergic neurons controlling paradoxical (REM) sleep. PLoS ONE 4:e4272

Sasaki T, Leutgeb S, Leutgeb JK (2015) Spatial and memory circuits in the medial entorhinal cortex. Curr Opin Neurobiol 32:16-23

Schmidt B, Marrone DF, Markus EJ (2012) Disambiguating the similar: the dentate gyrus and pattern separation. Behav Brain Res 226:56-65

Schwegler H, Lipp H-P, Van der Loos H (1981) Individual hippocampal mossy fiber distribution in mice correlates with two-way avoidance performance. Science 214:217-219

Segal M, Landis S (1974) Afferents to the hippocampus of the rat studied with the method of retrograde transport of horseradish peroxidase. Brain Res 78:1-15

Shahidi S, Motamedi F, Naghdi N (2004) Effect of reversible inactivation of the supramammillary nucleus on spatial learning and memory in rats. Brain Res 1026:267-274

Soussi R, Zhang N, Tahtakran S, Houser CR, Esclapez M (2010) Heterogeneity of the supramammillary-hippocampal pathways: evidence for a unique GABAergic neurotransmitter phenotype and regional differences: GABAergic and glutamatergic supramammillary-hippocampal pathways. Eur J Neurosci 32:771-785

Soussi R, Boulland JL, Bassot E, Bras H, Coulon P, Chaudhry FA, Storm-Mathisen J, Ferhat L, Esclapez M (2015) Reorganization of supramammillary-hippocampal pathways in the rat pilocarpine model of temporal lobe epilepsy: evidence for axon terminal sprouting. Brain Struct Funct 220:2449-2468

Swanson LW (1982) The projections of the ventral tegmental area and adjacent regions: a combined fluorescent retrograde tracer and immunofluorescence study in the rat. Brain Res Bull 9:321-353

Swanson LW (1998) Brain maps: structure of the rat brain. Elsevier, Amsterdam

Tye KM, Prakash R, Kim SY, Fenno LE, Grosenick L, Zarabi H, Thompson KR, Gradinaru V, Ramakrishnan C, Deisseroth K (2011) Amygdala circuitry mediating reversible and bidirectional control of anxiety. Nature 471:358-362

Ugolini G (2010) Advances in viral transneuronal tracing. J Neurosc Methods 194:2-20

Vertes RP (1992) PHA-L analysis of projections from the supramammillary nucleus in the rat. J Comp Neurol 326:595-622

Vertes RP (2015) Major diencephalic inputs to the hippocampus. Prog Brain Res 121:144

Vertes RP, Kocsis B (1997) Brainstem-diencephalo-septohippocampal systems controlling the theta rhythm of the hippocampus. Neuroscience 81:893-926

Vertes RP, McKenna JT (2000) Collateral projections from the supramammillary nucleus to the medial septum and hippocampus. Synapse 38:281-293

Wyss JM, Swanson LW, Cowan WM (1979) A study of subcortical afferents to the hippocampal formation in the rat. Neuroscience $4: 463-476$

Zhang N, Houser CR (1999) Ultrastructural localization of dynorphin in the dentate gyrus in human temporal lobe epilepsy: a study of reorganized mossy fiber synapses. J Comp Neurol 405:472-490

Publisher's Note Springer Nature remains neutral with regard to jurisdictional claims in published maps and institutional affiliations. 